\title{
1 Poly Ethylene Glycol (PEG)-Related Controllable and Sustainable Antidiabetic \\ Drug Delivery Systems
}

Yupeng Fu', Ying Ding ${ }^{\mathrm{a}}$, Litao Zhang ${ }^{\mathrm{a}}$, Yongmin Zhang ${ }^{\mathrm{a}, \mathrm{b}}$, Jiang Liu ${ }^{\mathrm{a} *}$, Peng Yu ${ }^{\mathrm{a} *}$

a China International Science and Technology Cooperation Base of Food Nutrition/Safety and Medicinal Chemistry, Key Laboratory of Industrial Fermentation Microbiology of Ministry of Education, Tianjin Key Laboratory of Industry Microbiology, College of Biotechnology, Tianjin University of Science \& Technology, 300457 Tianjin, China

${ }^{b}$ Sorbonne Université, CNRS, IPCM, UMR 8232, 4 Place Jussieu, 75005 Paris, France

*Corresponding authors e-mail address: liu.jiang@tust.edu.cn (Jiang Liu),yupeng@tust.edu.cn (Peng Yu)

\section{ABSTRACT}

Diabetes mellitus is one of the most challenging threats to global public health. To improve the therapy efficacy of antidiabetic drugs, numerous drug delivery systems have been developed. Polyethylene glycol (PEG) is a polymeric family sharing the same skeleton but with different molecular weights which is considered as a promising material for drug delivery. In the delivery of antidiabetic drugs, PEG captures much attention in the designing and preparation of sustainable and controllable release systems due to its unique features including hydrophilicity, biocompatibility and biodegradability. Due to the unique architecture, PEG molecules are also able to shelter delivery systems to decrease their immunogenicity and avoid undesirable enzymolysis. PEG has been applied in plenty of delivery systems such as micelles, vesicles, nanoparticles and hydrogels. In this review, we summarized several commonly used PEG-contained antidiabetic drug delivery systems and emphasized the advantages of stimuli-responsive function in these sustainable and controllable formations.

Keywords: PEG, drug delivery system, antidiabetic, stimulating responsive release, sustainable release 


\section{Content}

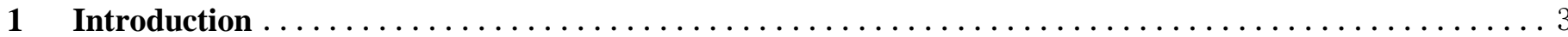

2 PEGylated micelles in antidiabetic treatments $\ldots \ldots \ldots \ldots \ldots \ldots \ldots \ldots \ldots \ldots \ldots \ldots \ldots \ldots$

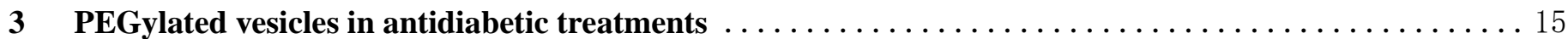

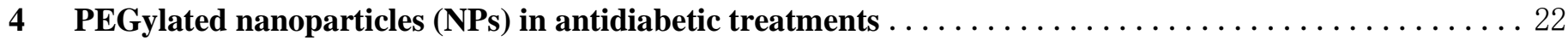

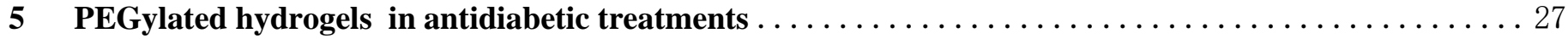

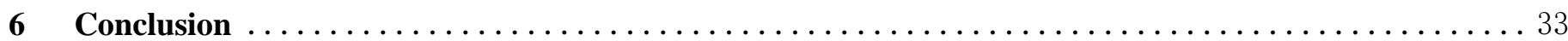

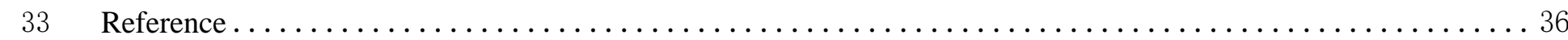




\section{Introduction}

Diabetes mellitus is a severe chronic metabolic disease which imposes enormous burdens both personally and socially ${ }^{1}$. It is estimated that 4 million people died of diabetes and its complications in 2017, which has caused vast economic losses ${ }^{2}$. Generally, diabetes is classified into three subtypes: type 1 diabetes mellitus (T1DM) is an autoimmune disease, induced by the destruction of pancreatic $\beta$-cells which produce insulin ${ }^{3}$, type 2 diabetes mellitus (T2DM) is generated by the insufficient insulin supply or the insulin resistance ${ }^{4}$, gestational diabetes mellitus (GDM) is a glucose intolerance which first diagnosed during pregnancy ${ }^{5}$. Among all these subtypes, T2DM is the biggest threat to public health, the figure for T2DM patients almost accounting for $90 \%$ of the total ${ }^{6}$. A wide range of diabetes therapies including behavioral $^{7}$, nutritional ${ }^{8}$, physical $^{9}$ and the most important, medicamentous therapies, have been adopted to alleviate diabetes and its complications.

Drug delivery systems (DDSs) plays a vital role in the clinical application of antidiabetic treatments since many antidiabetic drugs cannot achieve the ideal therapeutic effect without any assistance from a delivery system. Thus, appropriate DDSs are crucial in the designing of formulations. PEG-contained delivery systems are regarded as ideal options for the optimization of antidiabetic drug deliveries. PEG is a family of amphiphilic polymers ${ }^{10}$ that sharing the same skeleton of repeating ethylene glycol units $\left[\left(\mathrm{CH}_{2} \mathrm{CH}_{2} \mathrm{O}\right)_{n}\right]$ but with different molecular weights ${ }^{11}$. The most commonly used PEGylated reagents are the linear types of PEG chains. Except for the linear type, various shapes of PEG derivatives had been developed to meet different functions of specific attempts (figure 1). The derivation of PEG allowed more functionalization sites to be accessed, that broken through the limitations of the linear type PEG ${ }^{12}$, however, the derivation of PEG brought chemistry challenges in synthesis compared to the linear type PEG. Generally, PEGs have been extensively used in DDSs to ameliorate the physiochemical properties and bioactivity of various substrates ${ }^{13}$. 


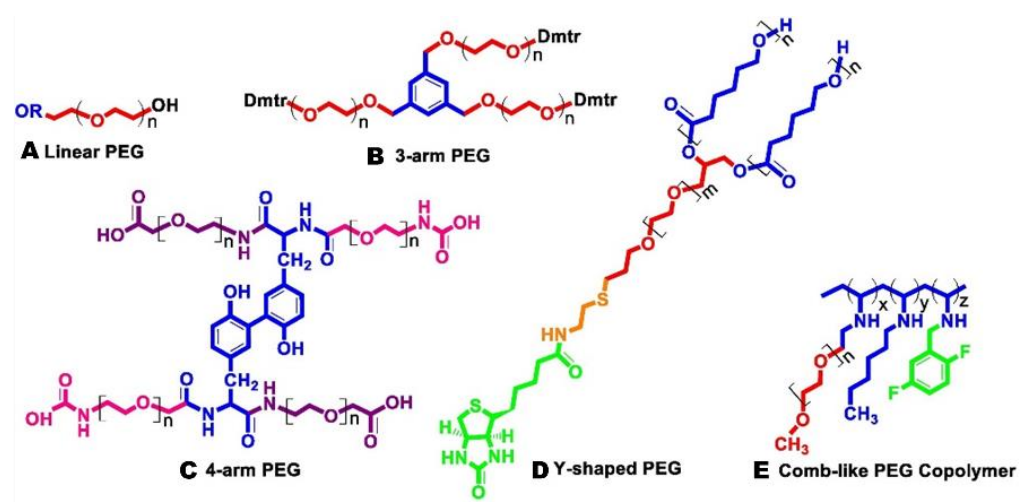

Figure 1. PEG and multiple derivatives obtained via different linking methods such as linear (A), 3-arm (B), 4-arm (C), Y-shaped (D) and comb-like polymers $(E)^{14}$.

PEG molecules consisting of the DDSs are able to shield DDSs and drugs by their long hydrophilic chains. This distinctive architecture generally renders the delivery particles four characters: 1) enhanced enzymatic stability; 2) expanded hydrodynamic volume; 3) reduced immunogenicity of the large biomolecules and 4) decreased possibility of the macromolecules aggregation. These properties can decrease the kidney clearance, prolong the internal lifespan and promote the stability of particles, which lead to improved therapeutic effects ${ }^{15}$. Owing to its unique structure, PEG endows natural or artificial materials with improved physiochemical properties and biodegradability ${ }^{16}$.

Besides, due to the absence of inherent bioactivity, antidiabetic PEGylated polymeric systems can provide optimization of delivery and release process in vivo such as the prolonged half-life time ${ }^{17}$. Diverse types of PEGylated antidiabetic DDSs including nanoparticles, microspheres, nanovesicles, micelles and hydrogels are able to fit novel administration of drugs like transdermal ${ }^{18}$ and oral administration ${ }^{19}$ of insulin and intelligent insulin injections ${ }^{20}$ which are able to overcome the disadvantages of traditional open-loop insulin delivery systems ${ }^{21}$. A number of studies have verified the advantages of PEGylated DDSs in diabetes treatments (table 1).

Table 1. Part of the PEGylated DDSs applied in antidiabetic treatment

Not applicable

$(\mathrm{N} / \mathrm{A})$
$N / A$

Glucose-responsive insulin release in vitro 
PEG/PNIPAM-P (Asp-co-AspPBA)/P

Insulin ${ }^{24}$

(Asp-co-AspGA-co-AspNTA) complex micelles

Insulin ${ }^{25}$

PEG- $b$-P(AA-co-APBA)/P(AA-co-AGA) micelles

Microsphere loaded with

Insulin 26

Insulin ${ }^{27}$

Insulin 28

Insulin ${ }^{18}$

Insulin ${ }^{29}$

Insulin ${ }^{30}$

DSPE-PEG-coated DPPC liposomes

Insulin ${ }^{32}$

Insulin ${ }^{31}$

Exenatide $^{33}$

Insulin ${ }^{34}$

Zn-insulin conjugates loaded by PLGA-PEG nanoparticles

HP-55-coated capsules loaded with

Insulin ${ }^{35}$

Plasmid DNA

encoding

GLP-1 $1^{36}$

$$
\text { conjugates }
$$

Insulin-deoxycholate composite micelles encapsulated by PCL-PEG-PCL vesicles nanoparticles

IgG Fc modified exenatide loaded by

PEG-PLGA nanoparticles nanoparticles

Linear PEI/plasmid DNA nanoparticles coated

by

DPPC/1,2-dimyristoyl-rac-glycero-3-methoxy
BALB/c male mice

Subcutaneous

STZ-induced mice

STZ-induced rats

STZ-induced rats

STZ-induced mice

STZ-induced mice

STZ-induced rats

STZ-induced mice

STZ-induced rats PEG-2000 (DMG-PEG)
Intraperitoneal

(i.p.) injection

N/A

N/A

S.c. injection

S.c. injection

Transdermal

administration

Transdermal

administration

Femoral

venous

cannula

S.c. injection

STZ-induced rats

$\mathrm{Db} / \mathrm{db}$ mice

$$
\text { N/A }
$$

N/A

Promote the renaturation of

DTT-induced aggregated insulin

Protease resistance, improved blood glucose regulation capacity

Enhanced glucose-responsive capacity

Reduced cytotoxicity, extended efficacy time

Improved encapsulation efficiency

Acid-responsive insulin release in vivo with extended blood glucose regulation time

Painless administration with glucose-responsive insulin release

Enhanced and stable blood glucose regulation ability with low hypoglycemia risk Improved incorporation efficiency and stability, more uniform particle size

Sustain therapeutic effect with same minimum blood glucose level compared with free insulin

Oral administration validity of insulin, prolonged plasma glucose regulation time

Obvious hypoglycemic effect

Oral administration compared with oral administration and SC injection of exenatide solution Enhanced entrapment capacity with small particle size, sufficient stability for

long-term storage

Oral administration validity of Oral insulin, improved cell uptake, administration extended efficacy time with stable hypoglycemic effect Obvious blood glucose

Balb/c mice and Oral $\mathrm{db} / \mathrm{db}$ mice administration regulation induced by highly expressed GLP-1 gene transfected by NPs 


\begin{abstract}
Exenatide $^{37}$ PEG- $b$ - $\left(\mathrm{PELG}_{50}-g-\mathrm{PLL}_{3}\right)$ nanoparticles

Zn-exenatide conjugates loaded by PEG-PLGA

Exenatide $^{38}$ nanoparticles with low molecular weight protamine as oral absorption promoter
\end{abstract}

SD rats and $\mathrm{db} / \mathrm{db}$ mice

STZ-induced rats

Female non-obese

diabetic mice

particles

Insulin ${ }^{40}$

Zn-insulin complexes loaded by PLA-PEG-PLA thermogel

STZ-induced SD rats

ICR mice and $\mathrm{db} / \mathrm{db}$ mice

ICR mice and SD rats hydrogel

SD rats system

Exenatide $^{43}$ Depot-gel-in-microsphere-in-Matrix-gel

Insulin ${ }^{44}$ 4-arm-PEG acrylic hydrogel

Insulin ${ }^{45}$ Semi-IPN chitosan-PEG-pAAm hydrogel

Insulin ${ }^{46}$
Arg-PEA/PEG-DA hybrid hydrogel withTD-1 as transdermal promoter
Oral

administration

S.c. injection

Prolonged plasma duration time and hypoglycemic effect

Oral administration validity, extended efficacy time, higher plasma maximum concentration, enlarged AUC (3.27 folds), enhanced bioavailability compared with s.c. administration

Oral administration

Oral administration

S.c. injection

S.c. injection

S.c. injection extended blood glucose regulation time, 7.6 folds larger AUC

Significant prolonged release time (46 days) with retained blood glucose regulation activity

Sensitive glucose-induced oxidation-degradation to achieve sustainable insulin release Sensitive glucose-responsiveness, improved swelling ratio, drug loading capacity and entrapment efficiency derived from the increased PEG ratio

Good biocompatibility, moderate and prolonged blood glucose regulation capacity 
are able to encapsulate drugs, including but not limited to small-molecule drugs, peptides, proteins, DNA, with high efficiency to achieve efficient administration ${ }^{47}$. Microneedles array is considered as an effective transdermal administration of insulin since its painless, low cost and convenient for self-administration ${ }^{48}$. Pharmacokinetically, transdermal administration like microneedles matrix is able to bypass the "first-pass" elimination and the reduced maximum blood drug concentration also could minimize the risk of side effects $^{49}$

In recent decades, long-acting formulations with stable and controllable drug release profile are gaining much attention. These "intelligent" formulations generally require an adjustable release process to get with the physiological or pathological changes (like blood glucose concentration fluctuation, temperature variation or oxidative conversion of circumstance) to achieve the optimal dosage distribution in the whole release process. For instance, a severe side effect of conventional insulin injections is the excessive hypoglycemia induced by the burst release of insulin in the blood, the efficacy time is also restricted. By contrast, the release profile of intelligent DDSs is more moderate and persistent. Thus, the responsiveness to physiological or pathological changes, in the other words, the stimuli-responsive capacity is one of the crucial features for sustain release formulations.

Specifically, glucose-responsive capacity, which is able to inhibit the burst release of drugs to prevent undesirable hypoglycemia events and prolong the plasma glucose regulation time, is important for intelligent formulations in antidiabetic treatments. The glucose-responsive capacity of PEG-based DDSs can be achieved by introducing glucose-sensitive functions. For example, phenylboronic acid (PBA) is able to endow PEG-based DDSs with glucose-responsive capacity. As scheme 1 demonstrated, there are two forms of PBA compounds in aqueous milieu ${ }^{50}$ : uncharged/relatively hydrophobic form and charged/relatively hydrophilic form. Since charged borate is capable of covalently forming a stable hydrophilic complex with glucose through the esterification between boronic acid and cis-diol group. This reaction induces the hydrophilic conversion of PBA-contained components in aqueous milieu (like blood), 
leading to the degradation of PBA-based micelles and the release of insulin loaded by micelles.
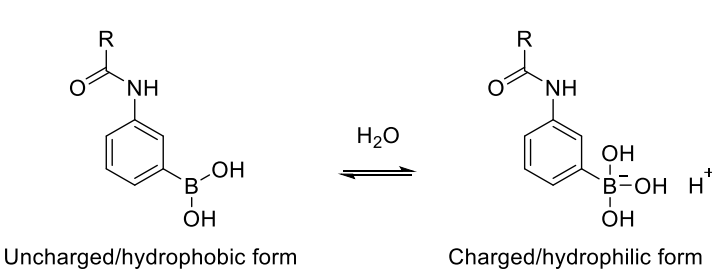

Charged/hydrophilic form

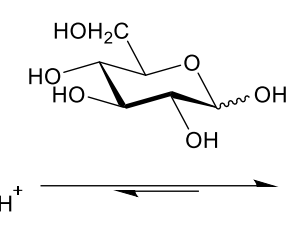

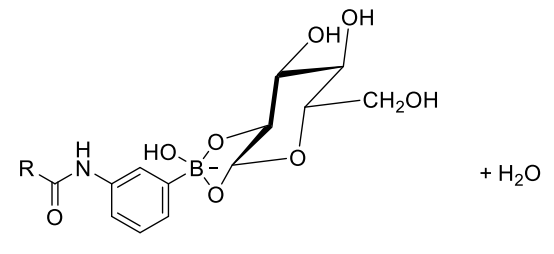

Stable hydrophilic complex

Scheme 1. The reversible formation of PBA-glucose complex

The earliest report of the reaction between glucose and PBA was reported by Kuivila, Henry G. et al. ${ }^{51}$.

As time goes by, plenty of studies detailed and optimized the application of PBA in the antidiabetic DDSs.

However, there are several challenges such as the discrepancy between apparent pKa for application and physiological $\mathrm{pH}^{52,53}$ and the insufficient sensitivity to the fluctuation of blood glucose level ${ }^{54}$ impede its further application. Therefore, a number of attempts have been deployed to form the accurate and adjustable glucose-responsive capacity to achieve controllable and sustainable drug release, providing more convenient formulations with better patient compliance.

Another case of stimuli-responsive capacity is glucose oxidase (GOx) which has been widely reported as a key component in the PEG-based DDSs. GOx is an enzyme that converts $\beta$-D-glucose and oxygen into gluconic acid and $\mathrm{H}_{2} \mathrm{O}_{2}{ }^{53}$. The intensive oxidation of $\mathrm{H}_{2} \mathrm{O}_{2}$ can induce many reactions including the dissociation of oxidation-sensitive materials. Thus, a number of DDSs choose $\mathrm{H}_{2} \mathrm{O}_{2}$ as the initiator of carrier degradation. However, the tissue inflammation induced by $\mathrm{H}_{2} \mathrm{O}_{2}$ is a challenge of $\mathrm{GOx}$-based glucose-responsive DDSs ${ }^{55}$. In general, the complexes of PEG and stimuli-responsive ingredients, because of their sustainable and controllable drug release capacity, are gradually being developed as a promising antidiabetic DDSs.

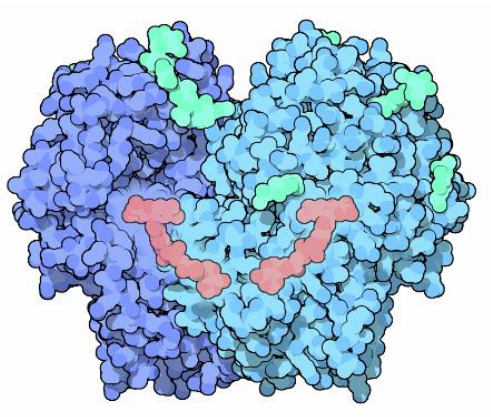


In this review, we discussed the application of PEG and PEGylated DDSs in the antidiabetic treatments antidiabetic DDSs, several instances are provided below with a detailed description.

\section{PEGylated micelles in antidiabetic treatments}

DDSs and stimuli-responsive capacity. In order to further illustrate the extensive applications of PEG in Among all kinds of micelles, the amphiphilic block copolymeric micelle draws much attention as DDSs ${ }^{56}$. Amphiphilic block copolymeric micelles are a series of thermodynamically stable colloidal dispersions consisting of amphiphilic block copolymers, being of diverse strengths such as stabilizing drugs, targeting delivery, enhancing cellular uptake $\mathrm{e}^{57,58}$. Three types of amphiphilic copolymeric micelles have been applied in the designing of DDSs: micelle-drug complexes are the composites of copolymeric micelles and drugs, micellar microcontainers trap drug molecules into their internal cavities to deliver them, and polyelectrolyte complexes are formed by the electronic interaction between cargoes and carriers, such as the conjugates of cationic block copolymers and polynucleotides ${ }^{59}$.

The amphiphilic block copolymeric micelles can be prepared by the self-assembling of the amphiphilic block copolymer chains which consist of two or more types of natural or synthetic polymers with different water affinity. The hydrophobic polymers consist of the internal side of the copolymeric chain and the hydrophilic polymers, like PEG, are placed on the other side to form external surface of micelles. In fact, PEG is considered as a popular ingredient to form amphiphilic micelles ${ }^{56}$. This unique architecture, widely known as the core-shell structure, allow micelles to deliver drugs with poor aqueous solubility in the water phase by loading them in the hydrophobic core of micelles ${ }^{60}$. In the PEG-contained amphiphilic block copolymeric micelles, the inner space of this spherical colloid encapsulates drugs and the outer PEG chains allow the micelle immune to unwanted results such as enzymolysis and aggregation ${ }^{61,62}$. 
copolymeric micelles. For instance, synthetic polypeptides have been applied because they are highly

biocompatible and biodegradable. Li Zhao and coworkers chose poly (L-glutamic acid) (PGA) to prepare monomethoxy PEG-b-poly (L-glutamic acid-co-N-3-L-glutamylamidophenylboronic acid) which designated as mPEG- $b$-P (GA-co-GPBA) micelles by modifying mPEG- $b$-PGA with 3-amino phenylboronic acid (APBA) to deliver insulin ${ }^{22}$. The whole synthetic route can be divided into two major steps (scheme 2).

Firstly, the copolymers mPEG- $b$-PGA were synthesized by the ring-opening polymerization (ROP) of y-Benzyl-L-glutamate-N-carboxyanhydride (BLG-NCA) which followed by the debenzylation. Secondly,

APBA molecules were coupled with the pendent carboxyl groups of GA units to afford copolymers mPEG- $b-\mathrm{P}$ (GA-co-GPBA). Afterwards, insulin was loaded into the hydrophobic core of the micelle via dialysis method in deionized water.

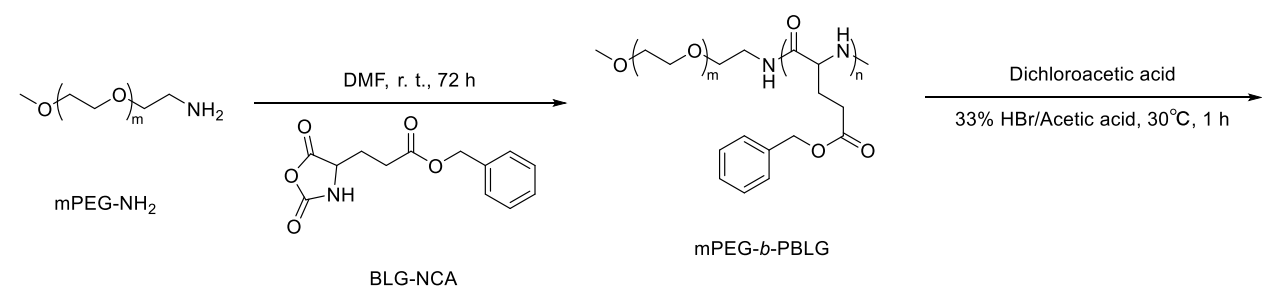

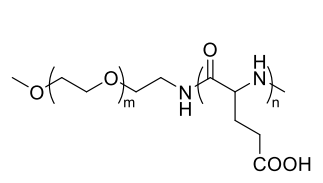

mPEG-b-PGA

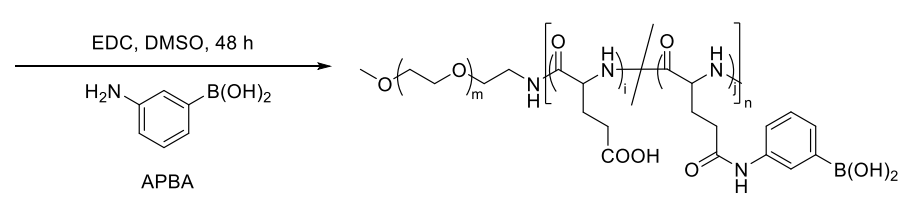

mPEG-b-P(GA-co-GPBA)

Scheme 2. Synthetic route of copolymers prepared by Li Zhao et al.

complexes. This hydrophilic variation allows the previously hydrophobic polymers to solve in the water

phase and the insulin loaded in advanced scatter in the local environment. Thus, these amphiphilic copolymeric micelles synthesized in the work of Li Zhao et al. exhibited glucose-responsive and adjustable drug release ability. As figure 3 exhibited, When the insulin-loaded micelles were added to phosphate buffer $(\mathrm{PB})$ without any glucose $(0 \mathrm{mg} / \mathrm{mL})$, insulin released very slowly: only $12.6 \%$ of the total were released within $3 \mathrm{~h}$. After the concentration of glucose increased to $3.0 \mathrm{mg} / \mathrm{mL}$, obvious release (37.7\%) 
was observed for the subsequent $3 \mathrm{~h}$. Switching the concentration back to $0 \mathrm{mg} / \mathrm{mL}$, insulin release was inhibited, only $7.2 \%$ amount was released in the following $3 \mathrm{~h}$. Then, as the concentration return to 3.0 $\mathrm{mg} / \mathrm{mL}$ again, release behavior was recovered, verified by $16.1 \%$ release of insulin within $3 \mathrm{~h}$.

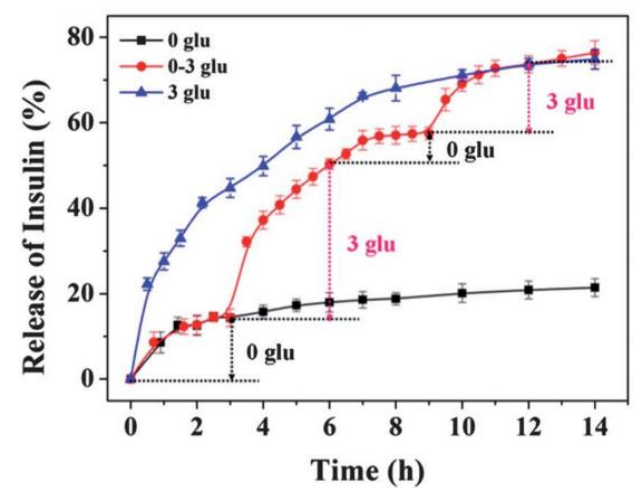

Figure 3. The release profile of glucose-responsive mPEG- $b-\mathrm{P}$ (GA-co-GPBA) micelles

In addition, no significant difference in circular dichroism (CD) spectra was observed between the released insulin and standard insulin sample, demonstrating that the preloaded insulin maintained its conformation after release. In vitro cytotoxicity investigation indicated the good biocompatibility of this copolymeric system.

This micelle system was prepared by Li Zhao et al., all components of the micelle are biodegradable and biocompatible, allowing safely elimination after the release of insulin. Besides, except for functioning as a hydrophilic group, PEG is also able to balance the increased immunogenicity and protect micelles from proteases $^{63}$ in these copolymeric systems. CD spectra verified the therapeutic validity of insulin loaded inside micelles. All these features could support the promising prospect of these copolymeric micelles as antidiabetic DDS.

Although polypeptides are of many advantages, a fatal demerit of these materials is the vulnerability to proteases which may weaken the protective effect of micelles on the loaded drug. Thus, Wu Gang and coworkers developed complex poly amino acids micelles with proteases resistance ${ }^{24}$. As figure 4 showed, PEG and poly ( $\mathrm{N}$-isopropylacrylamide) (PNIPAM) complex were employed as the composite shell, while 
(aspartic acid-co-aspartic acid glucosamine-co-aspartic acid nitrilotriacetic acid) designated as

$\mathrm{P}($ Asp-co-AspGA-co-AspNTA) functioned as the composite core. Insulin was connected to the divalent zinc ions which coordinated with NTA groups located on the P(Asp-co-AspGA-co-AspNTA) copolymeric chains. The glucose sensitivity of these composite micelles was derived from the PBA/GA complexation. PNIPAM played a crucial role in the protection of micelles from proteolysis by collapsing to form a hydrophobic shield. As expected, the resistance to proteases and improved blood glucose regulation capacity was observed. Under abnormally high blood glucose level, the interaction between glucose and PBA moieties gradually leads to the swelling and disassembly of complex micelles and the release of insulin.

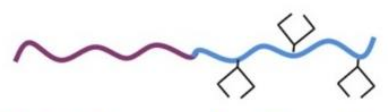

PNIPAM-b-P(Asp-co-AspGA-co-AspNTA)

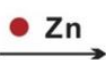

PNIPAM
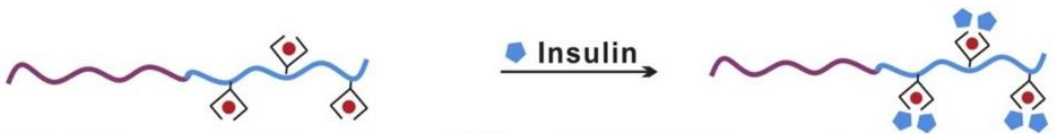

PNIPAM-b-P(Asp-co-AspGA-co-AspNTA[Zn])
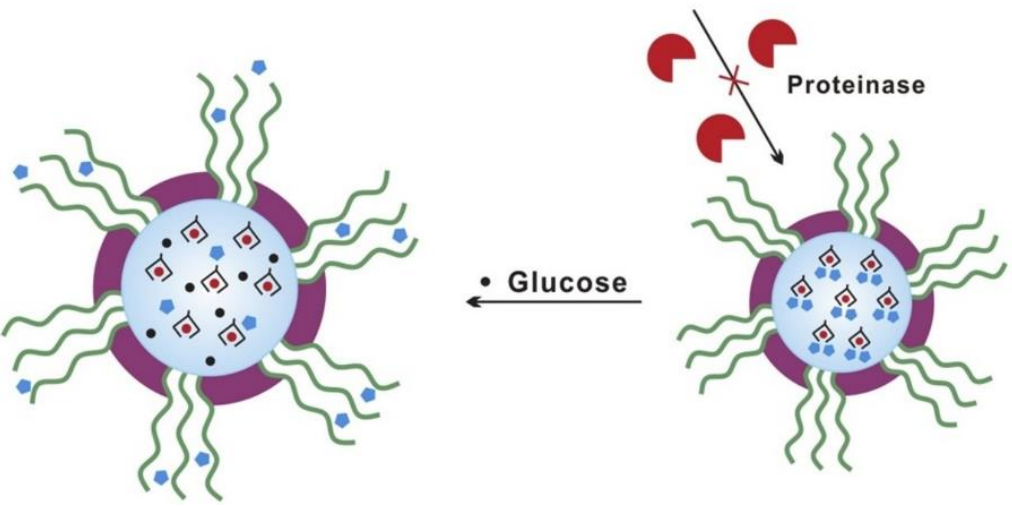

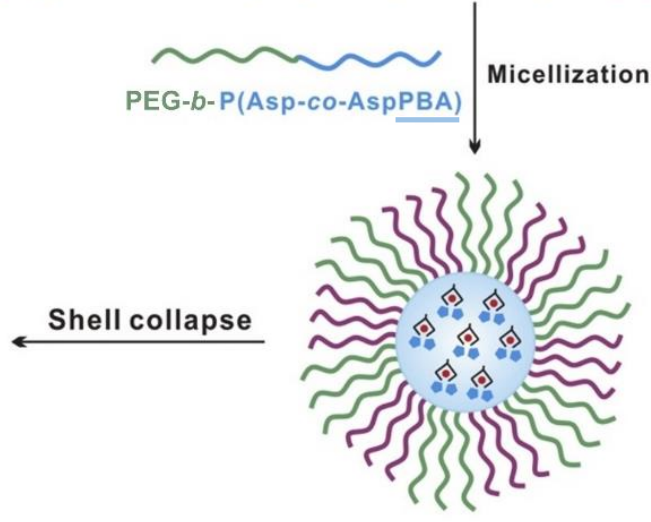

Figure 4. Schematic of the preparation and release process of complex micelles prepared by Wu Gang et al. ${ }^{24}$

Rujiang Ma et al. devised a type of glucose-responsive complex micelle consisting of block copolymer PEG-b-poly (acrylic acid-co-acrylamidophenylboronic acid) (PEG- $b$-P(AA-co-APBA)) and glycopolymer poly (acrylic acid-co-acrylglucosamine) $(\mathrm{P}(\mathrm{AA}-\mathrm{co}-\mathrm{AGA}))^{25}$. In this complex micelle system prepared by Rujiang Ma and coworkers, PEG chains on the outer layer functioned as a hydrophilic shell against aggregation. Another ingenious strategy is the introduction of glycopolymer: the most suitable $\mathrm{pH}$ for glucose-responsiveness is the apparent pKa of PBA (around $\mathrm{pH}$ 9) which is much higher than physiological $\mathrm{pH}$ (around 7.4), so the glucose sensitivity is restricted under physiological condition. Due to the complexation between PBA and glycopolymer, the apparent pKa of PBA was decreased and the glucose 
sensitivity was enhanced.

application. For example, insulin amyloid deposition has been found at the sites of frequent subcutaneous insulin injections ${ }^{64}$. This objectionable aggregation generally means the weakened hypoglycemic activity

Except for the enzyme degradation, undesirable aggregation is another obstacle to insulin delivery and of insulin ${ }^{23}$ and the increase of its immunogenicity ${ }^{65}$. Besides, these deposits also cause trouble in the production, storage and transportation of insulin ${ }^{66}$.

In recent decades, nanocage is gradually concerned as an alternative to traditional DDSs and PEGylation is regarded as an effective method of nanocage functionalization to obtain various advanced properties $^{67}$. A PEG-phosphatidylethanolamine (PEG-PE) micelle system was developed to achieve the reversion of insulin aggregation, inspired by the GroEL-GroES chaperonin system of Escherichia colf3. These diblock copolymeric micelles functioned as nanocages to concentration-dependently reverse the dithiothreitol (DTT)-induced insulin aggregation.

Insulin is a hypoglycemic protein with 51 amino acids, composed of two chains designated as A chain with 21 amino acids and B chain with 30 amino acids. These two chains are connected by two disulfide bonds between A and B chains. DTT can cut off these two disulfide bonds to afford separated unfolded peptide chains and the interaction between the hydrophobic parts of these chains finally produces the aggregations. Specifically, these nanocages were able to trap A and B chains of insulin cleaved by DTT, screening the interaction between their hydrophobic moieties which is the main factor of sedimentary formation. Besides, the separated $A$ and $B$ chains were able to reconnect with each other to afford native insulin with hypoglycemic activity. This process can be verified by the MALDI-TOF mass spectra and hypoglycemic effect in mice. CD spectroscopy suggested that PEG-PE micelles stabilized the secondary structure of native insulin, preventing chains from false folding.

Jun Wang and coworkers devised and prepared a type of cholic acid (CA)-PLGA- $b$-(polyethyleneimine (PEI)-PEG) micelles to load insulin on their surface through electrostatic interaction (figure 5$)^{26}$. An 
236 attractive point of this work reported by Jun Wang et al. is the combination of PLGA, PEI and PEG. PLGA has

237 been considered as an ideal material to build micro/nano structure for drug delivery ${ }^{68,69}$. However,

238 according to existing reports, the degradation of PLGA could produce an local acidic atmosphere ${ }^{70}$ which

239 may cause negative effects on the loading proteins and peptides but can be ameliorated by introducing

240 PEI into the delivery system ${ }^{71}$. However, the high cytotoxicity of PEI with large molecular weight (such as

$24125 \mathrm{kDa}$ ) derived from its excessive positive charge impedes its application ${ }^{72,73}$. Thus, PEG was introduced

242 into the system combining with low molecular weight PEI to ensure the safety of polymeric delivery system,

243 and the validity of these strategies have been confirmed ${ }^{73,74}$. Insulin was able to efficiently loaded on the

244 cationic polymeric micelles via the electrostatic force between the abundant cations on PEI-PEG layer and

245 insulin. Extended blood glucose regulation time was observed in in vivo investigation. 

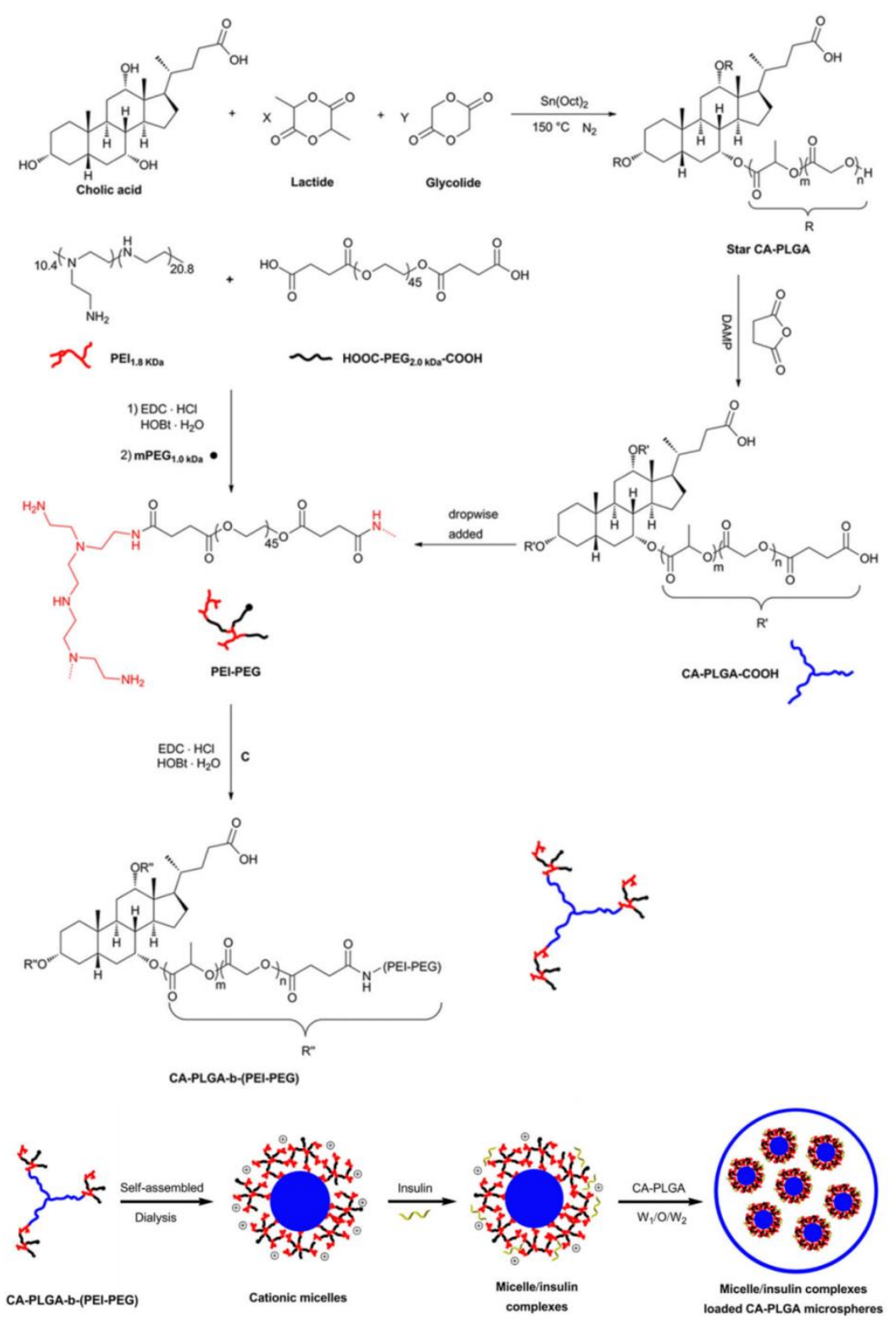

Figure 5. Preparation of the microsphere loaded with CA-PLGA- $b$-(PEI-PEG) micelles-insulin conjugates ${ }^{26}$.

\section{PEGylated vesicles in antidiabetic treatments}

Vesicles are a series of particles sharing a similar structure which consist of a lipid bilayer membrane and an internal hollow space separated from the outside. The bilayer membrane is composed of the hydrophilic "heads" which are generally forming the surface of the membrane and hydrophobic "tails" which are buried under "heads". Biologically, vesicles including liposomes and exosomes are of great 254 importance in the transportation, communication and other metabolic processes of various cells. The potential of vesicles as drug delivery systems has been widely reported ${ }^{75-77}$ such as paclitaxel-loaded 
257 circulation time ${ }^{78}$.

Inspired by bio-generated vesicles, synthetic ones are also have been developed to deliver drugs. For instance, inspired by native vesicles, Wanyi Tai et al. devised a biomimetic polymersome nanovesicle system with an acid-sensitive capacity ${ }^{28}$. This copolymeric vesicle employed PEG as the hydrophilic "heads" and ketal-modified polyserine (PEG-P (Ser-Ketal)) as the hydrophobic "tails" to form the bilayer membrane. Insulin, glucose oxidase (GOx) and catalase (CAT) were loaded in the hollow space of the vesicle. Drugs were well encapsulated while glucose molecules, due to their small size, were able to penetrate into the inside of the copolymer membrane. Afterwards, the interaction between glucose and GOx afforded gluconic acid and $\mathrm{H}_{2} \mathrm{O}_{2}$, leading to the local $\mathrm{pH}$ decrease. Meanwhile, $\mathrm{H}_{2} \mathrm{O}_{2}$ generated from the aforementioned process was converted to oxygen by CAT to avoid damaging other cellular components and the deactivation of $\mathrm{GOx}^{79-82}$. As the result of $\mathrm{pH}$ decrease, the ketals located on the polyserine segments of copolymers dissociated through acidic hydrolysis, causing hydrophilic conversion (figure 6 B) of entire copolymers in aqueous phase (like blood). Water-soluble copolymers without ketal moieties solved in the solution and the copolymeric membrane gradually fractured. Finally, insulin loaded in advance was released (figure $6 \mathrm{~A}$ ).

$\mathbf{A}$
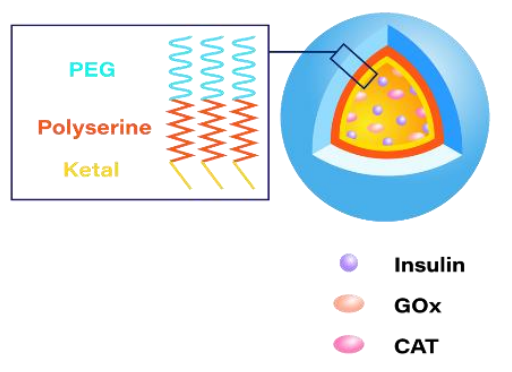

B

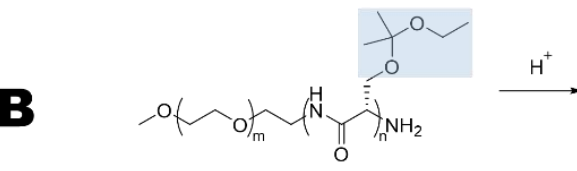

Amphiphilic PEG-Poly (Ser-Ketal)

Water soluble PEG-Polyserine

Figure 6. Schematic of the degradation of acid-sensitive diblock copolymer nanovesicles. 
The PEG-contained copolymeric nanovesicles were prepared by Wanyi Tai et al. exhibiting the glucose-responsive capacity. A low level of insulin release was observed under $100 \mathrm{mg} / \mathrm{dL}$ glucose or glucose-free condition in PBS buffer over $12 \mathrm{~h}$, compared with the rapid release under hyperglycemic condition. More importantly, with alternative conversion between normoglycemia and hyperglycemia every $2 \mathrm{~h}$, insulin release exhibited a pulsatile trend correspondingly (figure 7).

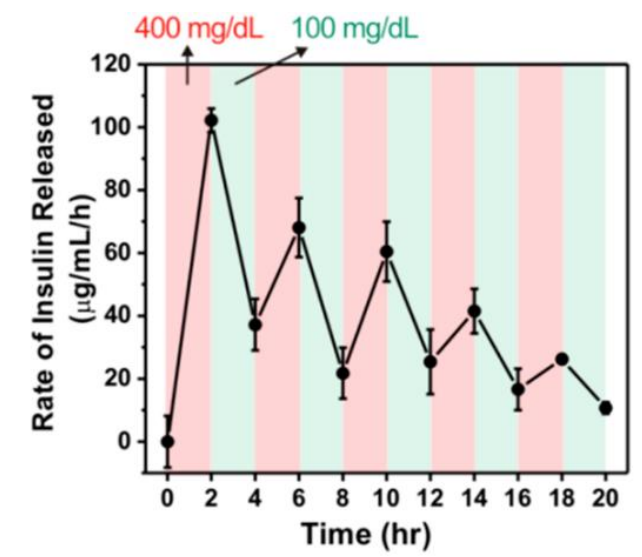

Figure 7. Pulsatile insulin release profile of PEG-P (Ser-Ketal) nanovesicles.

Difference up to 3-fold in release rate was observed when the glucose concentration altered. However, this trend gradually diminished as this circulation continued. A possible reason could be the gradual dissociation of vesicles and the leakage of enzymes.

Except for Wanyi Tai and coworkers, the applications of vesicles in the antidiabetic DDSs are also studied by others. Anna Kim et al. reported a distearoylphosphoethanolamine-PEG (DSPE-PEG)-coated 1,2-dipalmitoyl-sn-glycero-3-phosphocholine (DPPC) liposomes to reduce the uptake of liposome by reticuloendothelial system (RES) in parenteral administration ${ }^{30}$. After coated by DSPE-PEG, the liposomes were of more uniform size with enhanced aggregation resistance, and the circulation time was also extended by $1 \mathrm{~h}$.

In addition to individually applicated, vesicles are also able to combine with other delivery systems. Rachna Rastogi et al. developed a poly (caprolactone)-PEG-poly (caprolactone), which designated as PCL-PEG-PCL, copolymeric vesicle system to encapsulated insulin-deoxycholate composite micelles ${ }^{27}$. 
10-50\%. Burst insulin release was weakened and the efficacy time was prolonged by $2 \mathrm{~h}$, but the increased

hydrophobicity of the delivery system exerted a negative influence on its pharmacological effects which

emphasize the critical role of equilibrium between hydrophilicity and lipophilicity.

Another case is microneedles-vesicles composite delivery system. Microneedles (MNs) have been

introduced to antidiabetic DDSs to achieve painless transdermal administration which is able to promote patient compliance due to their unique properties ${ }^{53}$. MNs (figure 8) have been extensively explored for the transdermal administration of various substances such as small molecule drugs ${ }^{83}$, proteins ${ }^{84}$ and particles ${ }^{85}$, and diverse type of MNs including hollow, solid, coated, dissolving and hydrogel forming ${ }^{49}$ are also developed by various materials. The application of MNs in antidiabetic DDSs have been systematically reported $^{48,49}$
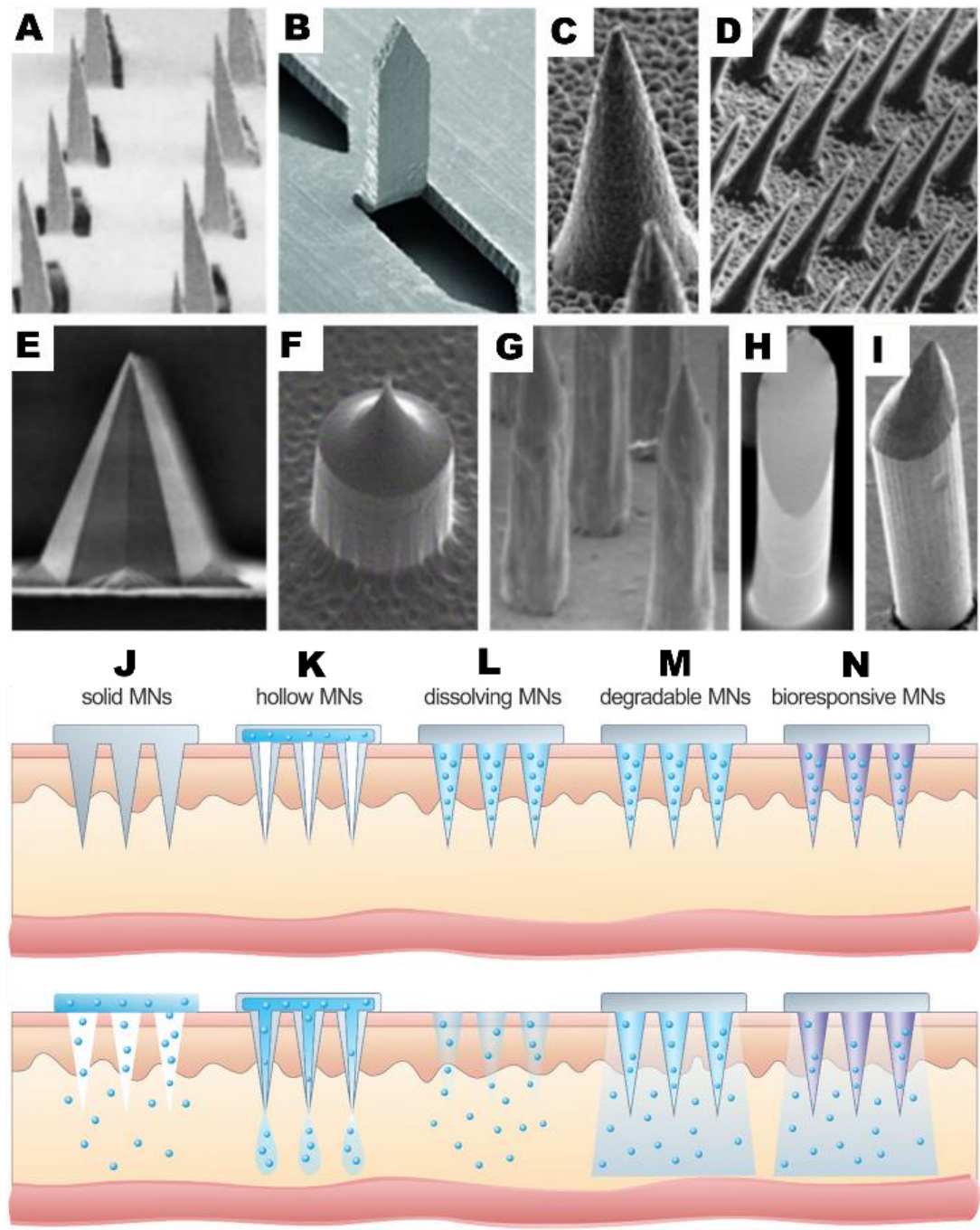

Figure 8. Images of different shapes of MNs (A-I) ${ }^{48}$ and different type of MNs including solid $\mathrm{MNs}(\mathrm{J})$, hollow MNs (K), 
The painless and efficient transdermal delivery capacity of MNs is derived from their unique architecture. As figure 9 displayed, stratum corneum (SC) with a thickness of 10-15 $\mu \mathrm{m}$ is considered as the main obstacle for transdermal administration. According to reports, only sufficiently lipophilic substances with molecular weight lower than $500 \mathrm{Da}^{87}$, which is too small for most of the vesicles, could diffuse into SC. As the result, common transdermal administration is restricted for vesicles. Fortunately, nerves are located the SC without or slightly touching nerves, inducing little or no pain. Besides, compared with conventional transdermal formulations which are highly restricted by the diffusion limit of SC, MNs are able to efficiently deliver the diver types of drugs without SC impedance. The introduction of MNs allow the vesicles which are too large to penetrate SC by self-diffuse to cross this screen with high efficiency, and glucose-responsive vesicles endow formulations with sustainable and controllable release, achieving optimal dosage distribution. The MNs-vesicles complex delivery systems integrate both the efficient transdermal administration and glucose-responsive capacity.

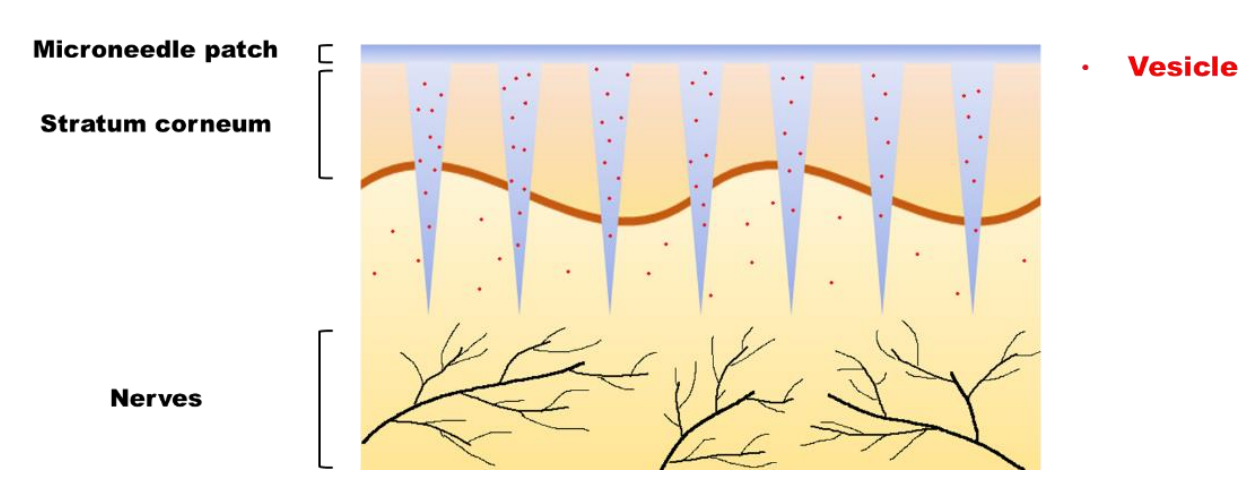

Figure 9. The transdermal administration of vesicles performed by microneedles array. a few hundred microns below $\mathrm{SC}^{88}$. Thus, the painless administration can be achieved through bypassing

transdermaladministration and glucose-responsive capacity.

Xiuli Hu et al. integrated self-assembling amphiphilic block copolymeric vesicles which were composed of PEG, polyserine and phenylboronic ester (PBE) with microneedles array to form a MNs/vesicles complex delivery system to achieve sustainable and controllable release ${ }^{18}$. Cross-linked hyaluronic acid (HA) was 
vesicles-loaded MNs array. Figure $10 \mathrm{~B}$ exhibits that excessive blood glucose concentration causes the disassociation of vesicles and the consequent insulin release. Figure $10 \mathrm{C}$ displays that the origin of $\mathrm{H}_{2} \mathrm{O}_{2}$ which was directly responsible for the dissociation of vesicles is the interaction between GOx encapsulated in the vesicles and the glucose penetrating into the inside of vesicles.

A

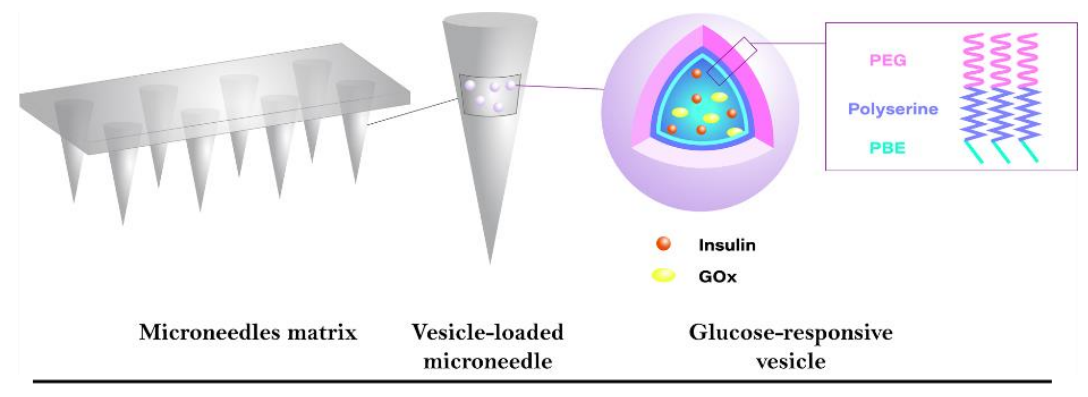

B

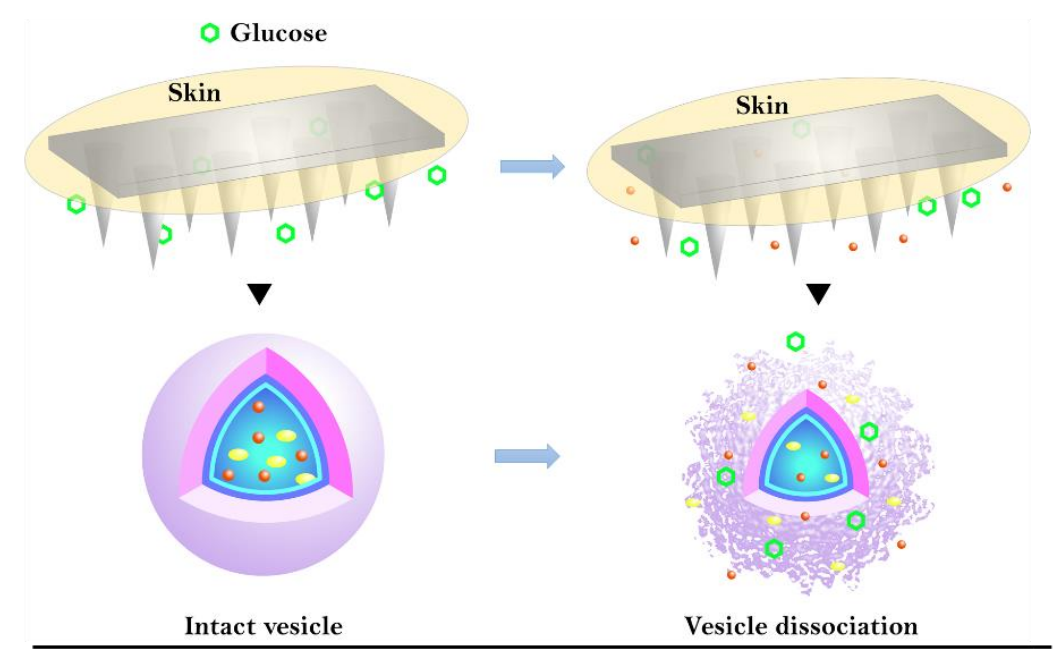

being able to solve in the aqueous phase. Consequently, gradual degradation of vesicles led to the release of preloaded insulin. Xiuli Hu et al. adopted the strategy mentioned above to endow the vesicles with 

including inflammation. interval.

and afford $\mathrm{H}_{2} \mathrm{O}_{2}$ which is responsible for the disassembly of vesicles. These vesicles were encapsulated into microneedles formed by cross-linked HA, since HA is highly biocompatible with appropriate stiffness to penetrate skin. Once the plasma glucose level was abnormally high, surplus glucose penetrated the HA membrane into the inside of vesicles, reacting with $\mathrm{GOx}$ to yield $\mathrm{H}_{2} \mathrm{O}_{2}$, leading to the dissociation of vesicles and the release of insulin loaded in advance. The in vivo influence was carefully evaluated: the biocompatibility of this formulation was acceptable, along with the negligible hypoglycemic risk. Rapid glucose-responsive insulin release was observed under hyperglycemia and once reaching normoglycemia, the release rate was restrained. On the one hand, the glucose-sensitive drug release capacity of this MNs loaded with mPEG-b-P(Ser-PBE) vesicles reduce the risk of undesirable side effects such as hypoglycemia and potential damage induced by $\mathrm{H}_{2} \mathrm{O}_{2}$ to cells; on the other hand, this $\mathrm{MNs}$ allows the optimized dosage distribution for drug release, significantly prolonged blood glucose regulation time and administration

In another case of the composite MNs delivery system reported by Jicheng Yu and co-workers ${ }^{29}$, a type of MNs matrix containing hypoxia and $\mathrm{H}_{2} \mathrm{O}_{2}$ dual-sensitive vesicles was developed based on a similar strategy. The loaded copolymeric vesicles, designated as PEG-P (Ser-S-NI) vesicles, were composed of PEG and polyserine modified by 2-nitroimidazole through thioether bridge. The $\mathrm{H}_{2} \mathrm{O}_{2}$ and hypoxia-responsive capacity derived from the thioether and 2-nitroimidazole respectively. The thioether was transformed to sulfone after reacting with $\mathrm{H}_{2} \mathrm{O}_{2}$, this oxidation leading to the hydrophilic conversion of polymer ${ }^{89,90}$. Simultaneously, 2-nitroimidazole converted to hydrophilic 2-aminoimidazole under hypoxia condition which was mediated by the transformation from glucose to gluconic acid ${ }^{91,92}$. In this composite system, the hypoxia-responsive 2-nitroimidazole parts enhance the glucose sensitivity of delivery systems, and the $\mathrm{H}_{2} \mathrm{O}_{2}$-responsive thioether parts consume excessive $\mathrm{H}_{2} \mathrm{O}_{2}$ generated by $\mathrm{GOx}$, avoiding negative effects 


\section{PEGylated nanoparticles (NPs) in antidiabetic treatments}

The benefits of PEGylation to NPs have been systematically summarized ${ }^{93}$ and verified by many studies $^{20,31-34,36,38,39,94-96}$. Basically, PEG as ingredients could resolve the instability of insulin in the harsh formulation conditions, while poly ( $D$, L-lactide-co-glycolide acid) (PLGA) is a type of polymer that has already been successfully applied in biomacromolecule delivery ${ }^{69}$. Yusuf Haggag and coworkers employed double emulsion technique to prepare a series of NPs consisting of poly (D, L-lactic-co-glycolic acid) (PLAG)-PEG diblock polymers to optimize the entrapment efficiency of insulin ${ }^{31}$. According to the experimental results, part of PLGA-PEG polymers via homogenized insulin-loading method optimized their entrapment efficiency and release kinetics of insulin. The in vivo studies revealed the aggregation resistance, improved stability and the maximum retention of hypoglycemic bioactivity of insulin during the whole release process. Moreover, the PLGA-PEG diblock copolymeric NP system devised by Yusuf Haggag et al. exhibited a marked insulin sustainable release manner.

A similar PLGA-PEG NPs system was designed by Sunandini Chopra and coworkers ${ }^{34}$. The difference was that they added zinc ions to insulin to form the insulin-Zn hexamers via the chelation first, then co-assembled with PLGA-PEG under a carefully adjusted $\mathrm{pH}$ nanopercipitation condition to form NPs. Finally, after a PBS washing process, the insulin-Zn PLGA-PEG NPs were afforded (figure 11). NPs prepared via this complexation between zinc ion and insulin exhibit significant improvement (about 10-fold) in insulin loading capacity.

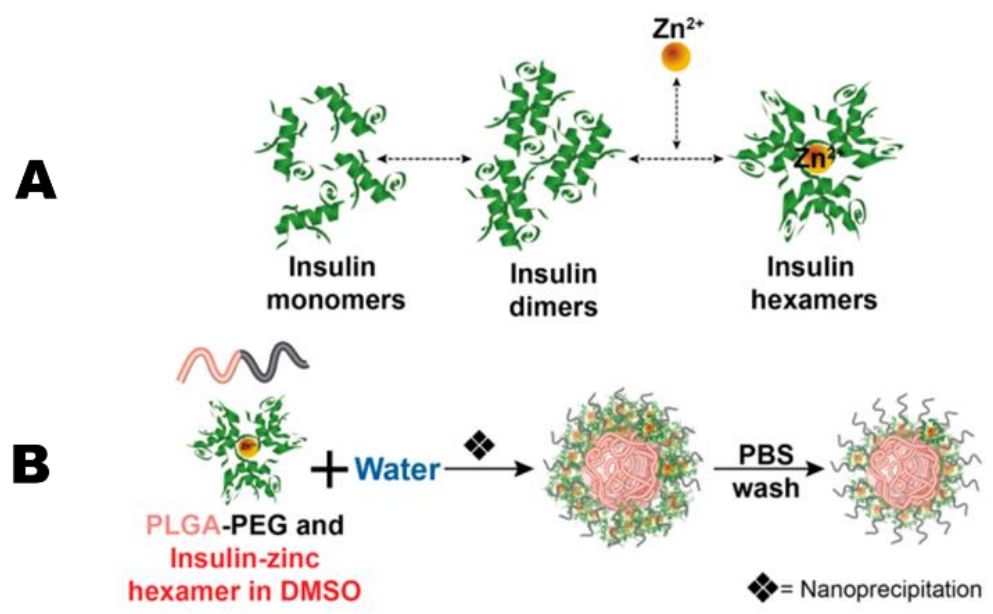

Figure 11. Schematic of the preparation of insulin-Zn complex and the NPs developed by Sunandini Chopra and 
In the work of Shelesh JAIN and Swarnlata SARAF, PEG significantly extended the efficacy time of

types of NPs designated as RPGNP1 and RPGNP2 were prepared in this work, based on PLGA and mPEG-PLGA copolymer respectively. The loading capacity of pure PLGA NPs was $58.7 \pm 1.3$ and the mPEG-PLGA was less than the former, being $45.8 \pm 1.2$. In vitro release experiments indicated that both of these two formulations experienced an initial burst release and a following sustained release process. However, different trends were observed in the in vivo investigations. The blood glucose level of diabetic rats treated by PLGA NPs decreased within the first $24 \mathrm{~h}$ and began to climb afterward, while the hypoglycemic effect of mPEG-PLGA NPs maintained over $7 \mathrm{~d}$ (figure 12). A possible reason could be the different affinity to liver macrophages: RPGNP2 were hardly identified by liver macrophages due to their PEG structure and able to retain for a relatively long time, while RPGNP1 could be rapidly identified and neutralized by liver macrophages without the PEG sheltering effect.

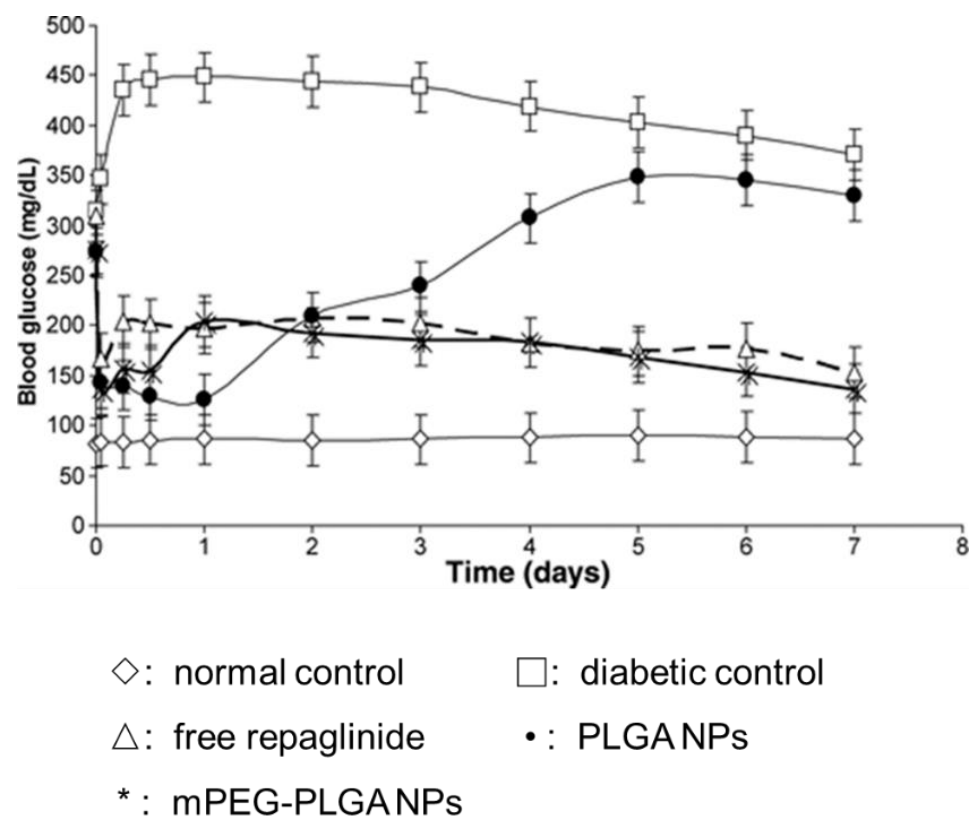

Figure 12. Blood glucose level of different in vivo experimental groups in the work of Shelesh JAIN and Swarnlata SARAF.

Yanan Shi et al. prepared a different type of PLGA-PEG NPs, with Fc modified for the oral delivery of 
exenatide $^{33}$. Exenatide is a GLP-1 analogue with 39 amino acids. GLP-1 is a versatile peptide generated by

the proglucagon gene, mainly produced by the intestinal L-cells ${ }^{97}$. GLP-1 have been widely concerned as incretin hormones to treat diabetes ${ }^{98,99}$. However, a fatal demerit of natural GLP-1 is extremely short lifespan. Due to the high affinity with plasma dipeptidyl peptidase 4 (DPP-4), GLP-1 can be neutralized within $2 \min ^{97}$. By contrast, the half-life of exenatide is $2.4 \mathrm{~h}^{100}$. The Fc-targeted NPs could bind to Fc receptor which is expressed on the epithelial cells in the small intestine that could help the absorption of NPs. The in vitro and in vivo studies verified the better cell uptake and hypoglycemia maintaining performance. Their group also developed low molecular weight protamine (LMWP)-contained PEG-PLGA NPs for the oral delivery of Zn-exenatide complexes ${ }^{38}$. In this delivery system, the LMWP could increase the penetrability of the whole delivery system which was confirmed by the cellular uptake experiment compared with the pure copolymeric delivery system, the bioavailability also exhibited great improvement. Similar to the strategy of Jun Wang et al., using the electrostatic interaction to connect drugs and carriers, Fei Tong prepared PEG- $b-\left(\mathrm{PELG}_{50}-g-\mathrm{PLL}_{3}\right)$ polymeric NPs to carry exenatide via electrostatic force between the negative exenatide molecules and positive polymers under $\mathrm{pH} 7.4$ (figure $13 \mathrm{~A})^{37}$. As Fei Tong reported, the loading efficiency of these PEG- $b-\left(\mathrm{PELG}_{50}-g-\mathrm{PLL}_{3}\right)$ NPs on exenatide was $12.11 \%$. The cumulative release profile indicated that the release of exenatide displayed a very stable and sustainable manor (figure $13 \mathrm{~B}$ ), and the observation of blood glucose level revealed a significantly prolonged hypoglycemic activity (figure $13 \mathrm{C}$ ). Besides, alleviated diabetic nephropathy was also observed in this study. 


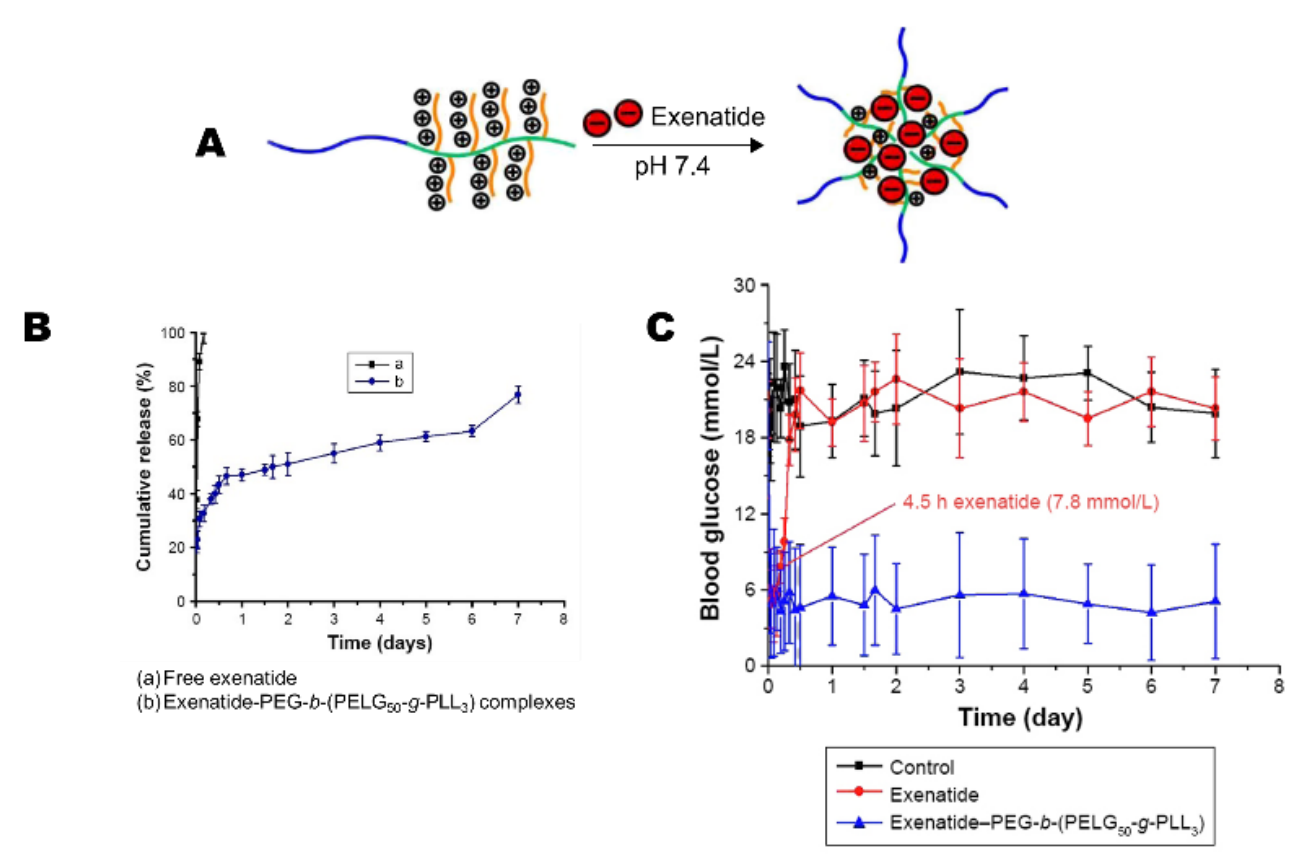

Figure 13. A: formation of exenatide-loaded PEG-b-(PELG50-g-PLL3) NPs; B: cumulative release profile of NPs; $C$ : blood glucose concentration of groups treated differently ${ }^{37}$.

In addition to delivery GLP-1 analogues, according to Tianqi Nie et al. reported, plasmids DNA encoding GLP-1 were also able to be delivered by NPs ${ }^{36}$. DNA formulations are of various advantages but the rapid degradation of pure DNA and the poor ability to cross the mucus layer in the gastrointestinal tract are still the main challenges in their applications ${ }^{36,101}$. PEI has already been reported widely as an efficient transfection compounds ${ }^{102-104}$. As figure 14 showed, in the work of Tianqi Nie et al., plasmid DNA encoding GLP-1 was complexed with linear PEl to form NPs. Afterwards, DPPC and 1,2-dimyristoyl-rac-glycero-3-mPEG-2000 (DMG-PEG) were adopted to coating NPs, forming linear PEI/plasmid DNA NPs with a hydrophilic and electrostatically neutral shell which could benefit the penetration process of mucus layer since its highly hydrophilic with abundant cationic charges via an exquisite method named as flash nanocomplexation (FNC). 
Figure 14. Preparation of linear PEI/plasmid DNA NPs coated by DPPC/DMP-PEG via FNC technology and the expected penetration process through the gastrointestinal tract ${ }^{36}$.

In vitro investigations indicated the obviously reduced cytotoxicity induced by the DPPC/DMG-PEG shell and the high transfection efficiency in A549 and HeLa cell lines. In vivo experiments pointed out the high efficiency of transfection in the lung and liver, while the good biocompatibility was verified by ameliorated liver damage observed in the toxicity evaluation. Besides, stable expression of GLP-1 resulted in sufficient blood glucose regulation capacity, confirming the potential of the FNC-prepared linear PEI/plasmid DNA NPs coated by DPPC/DMP-PEG.

In order to further improve the physiochemical properties to promote the insulin delivery capacity of PEGylated NPs, pharmaceutical spray drying was adopted in the preparation of NPs (figure 15). Various benefits to drug encapsulation of this formulation technology have been reported ${ }^{105,106}$.
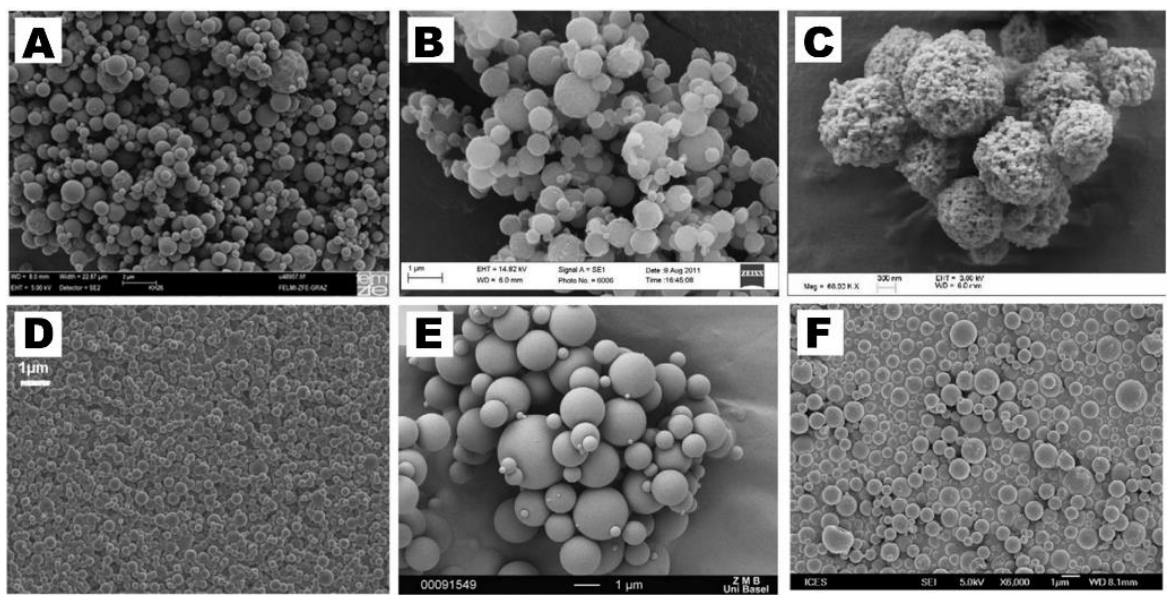

Figure 15. SEM images of several NPs produced by spray drying collected by Cordin Arpagaus et al. ${ }^{106}$ 
Spray freeze drying (SFD) is deriving from the general spray drying have been applied to prepare NPs as insulin carriers $^{107}$. In this technology, the notable advantage is it can maximum retain the insulin's bioactivity due to the drying process under low temperature.

Fei $\mathrm{Yu}$ et al. toke the advantage of SFD to form a type of hydroxypropyl methylcellulose phthalate-coated hard gelatin capsules (HP55) loaded with mono-dispersed microparticles containing insulin-loaded PLGA-lipid-PEG nanoparticles (designated as micro-particles@INS-PLGA-lipid-PEG NPs) for oral administration of insulin (figure 16), exhibiting excellent entrapment efficiency (92.3\%), much more cellular uptake efficiency than the naked insulin and prolonged decreasing blood glucose level in diabetic mice with oral administration ${ }^{35}$. This kind of NPs combine the advantages of both polymeric NPs and liposomes $^{108}$. Three different functional domains constitute the NPs: a hydrophobic PLGA core as the insulin carrier, an amphiphilic middle layer composed of soybean phosphatidylcholine (SPC) promotes the delivery efficiency and a PEG shell provides physiological stability. Gradient insulin release and elimination were observed after oral administration of the prepared LPNs compared with the rapid release rate induced by subcutaneous injection.

Sampath Malathi and coworkers prepared a series of $D$ - $\alpha$-tocopherol PEG 1000 succinate (TPGS)-emulsified PEG-capped PLGA NPs (ISTPPLG NPs) for insulin delivery via oral administration ${ }^{32}$. The rat trials suggested that the ISTPPLG NPs could successfully decrease the serum glucose level and last for $24 \mathrm{~h}$. Notably, the ISTPPLG NPs showed a regenerative effect of the liver, kidneys and pancreas on diabetic rats compared to normal control rats.

\section{PEGylated hydrogels in antidiabetic treatments}

Hydrogel is a type of water-swollen networks mainly consisting of polymer ${ }^{109}$, and PEG is a widely used 
ingredient for hydrogels which have been extensively studied to achieve controllable and sustainable antidiabetic drug delivery. Plenty of studies report the DDSs based on hydrogels ${ }^{41-46,110-115}$. Basically, a wide range of substrates can be loaded in the PEG-based hydrogel systems due to their unique structural characters: the highly customizable block length of PEG and other components of hydrogel allow the adjustment of equilibrium between hydrophobicity and hydrophilicity, enabling the universality of different molecules ${ }^{116}$. Another advantage of copolymeric hydrogels is the diverse stimuli-responsive capacity. Thermosensitive, $\mathrm{pH}$-responsive hydrogels and many other species have been developed and applied in DDSs ${ }^{117}$. Besides, varying the ratio of different components and combining two or more distinct copolymers also could improve loading capacity. Some examples of diabetes treatment are listed below. (figure 16). The lower critical solution temperature (LCST) is the lowest temperature that the polymer remains soluble in aqueous solvent which is injectable. Once beyond the LCST, polymer will transfer to solid gel state to form local drug storage.

Thermosensitive hydrogels are the most investigated stimuli-sensitive species ${ }^{118}$, already been applied in the delivery of biomacromolecules ${ }^{119}$. Phase diagrams reveal the conversion between liquid and solid

Figure 16. Phase diagrams mark the lower critical solution temperature (LCST) and the upper critical solution temperature (UCST) as the temperature barriers between monophasic and biphasic mixture ${ }^{117}$.

Up to date, thermosensitive hydrogels have already been adopted to deliver antidiabetic drugs. Yipei Chen et al. described a series of injectable thermosensitive hydrogels to achieve sustained release of 
497 ( $\varepsilon$-caprolactone-co-glycolic acid) (PCGA-PEG-PCGA), obtained via typical ROP, being of similar molecular

weights while the ratio of $\varepsilon$-caprolactone-co-glycolide was various. As reported, these biocompatible copolymers were able to convert to gel from aqueous solution as the ambient temperature increased, which means that copolymers loaded lira were injectable under room temperature, once these thermosensitive materials enter the body, they could form stable hydrogels in situ to build drug storages and release pre-encapsulated lira continuously. This copolymer hydrogel formulation fulfills the demand of both sustain drug delivery and painless administration. Meanwhile, pancreatic function benefits were observed, indicating the considerable clinical value of these thermosensitive hydrogels.

The loaded drug, lira, is a palmityl-acylated derivative of GLP-1. As figure 17 exhibited, the replacement of lysine with arginine at position 34 and the linkage of a 16-carbon fatty acid at position $26^{120}$ impede the degradation induced by DPP- $4^{121}$, extending the half-life of lira (11-13 h) compared with the unmodified GLP-1 (no more than 2 min) ${ }^{122}$. Besides, various pharmacological activities including the normalization of serum glucose level, the regulation of cardiovascular situation ${ }^{123}$ and the promotion of $\beta$-cell proliferation ${ }^{124}$ have been reported.

$7 \quad 9$

His-Ala-Glu-Gly-Thr-Phe-Thr-Ser-Asp-Val-Ser-Ser-Tyr-Leu-Glu-Gly-Gln-Ala-Ala-Lys-Glu - fatty acid (palmitoyl) Gly-Arg-Gly-Arg-Val-Leu-Trp-Ala-Ile-Phe-Glu 36

Figure 17. Structure of liraglutide ${ }^{125}$.

A PLGA-PEG-PLGA triblock copolymeric thermosensitive hydrogel was reported ${ }^{42}$ as the carrier of another versatile GLP-1 receptor agonists lixisenatide (lixi) ${ }^{126-128}$ since the electronic interaction between lixi and PLGA-PEG-PLGA benefit the stability of lixi. Except for PLGA-PEG-PLGA, PCGA-PEG-PCGA copolymers and the mixture of these two copolymeric systems were also investigated. According to the literature, blending hydrogels are of more stable degradation performance in vivo than PLGA-PEG-PLGA or PCGA-PEG-PCGA hydrogel alone. Most of all, as the pharmacokinetic study of mixture suggesting, 
remarkable pharmaceutical improvements were observed including prolonged half-life time and enlarged

AUC (table 2).

Table 2. Pharmacokinetic investigation unfolded the enhancement of hydrogel formulation ${ }^{42}$.

\begin{tabular}{cccccc}
\hline sample & $C_{\max }{ }^{\mathrm{a}}(\mathrm{ng} / \mathrm{mL})$ & ${ }^{\mathrm{b}} \mathrm{T}_{\max }(\mathrm{h})$ & ${ }_{\mathrm{t}_{1 / 2 z}}(\mathrm{~h})$ & $\mathrm{AUC}_{(0-\text { last })}(\mathrm{h} \mathrm{ng} / \mathrm{mL})^{\mathrm{d}}$ & $\mathrm{MRT}^{\mathrm{e}}$ \\
\hline free Lixi & 106.4 & 1.0 & 2.2 & 378.9 & 2.8 \\
Lixi/Gel & 24.5 & 0.5 & 30.3 & 2891.6 & 94.6
\end{tabular}

a: maximum plasma concentration; b: time required to reach the maximum plasma concentration; c: plasma half elimination time; $d$ : area under the curve; e: mean retention time.

Divya Sharma and Jagdish Singh prepared a PLGA-PEG-PLGA copolymeric hydrogel system to load chitosan-zinc-insulin complexes ${ }^{40}$. The complex of insulin and zinc and the application of oleic-modified chitosan polymer significantly stabilized insulin and its distribution process among this thermosensitive injectable hydrogel. In vivo investigation indicated the more stable blood concentration of insulin and the prolonged blood glucose regulation time compared with free insulin.

Mei Zhang and coworkers $^{44}$ reported an oxidation-responsive hydrogel polymerized by

GOx were loaded into the hydrogel network. 

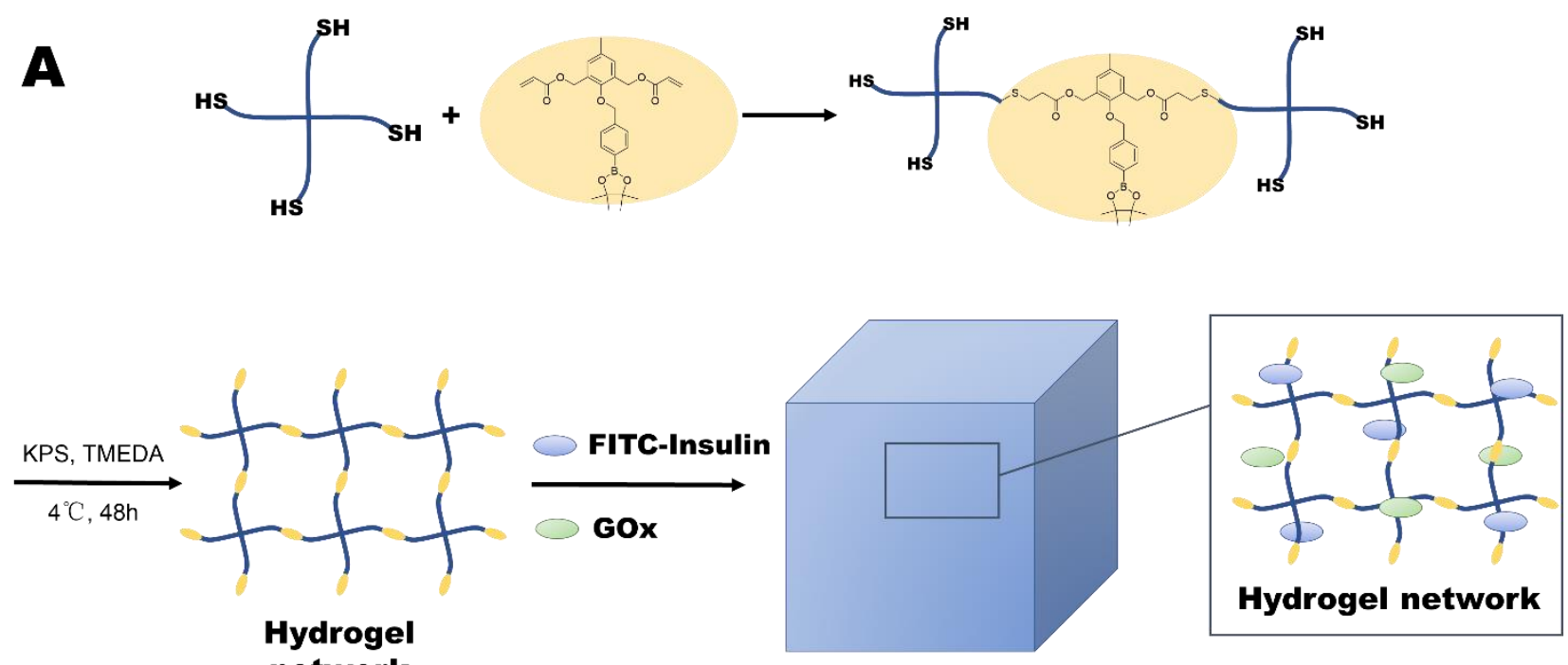

\section{FITC-insulin/GOx-loaded hydrogel}
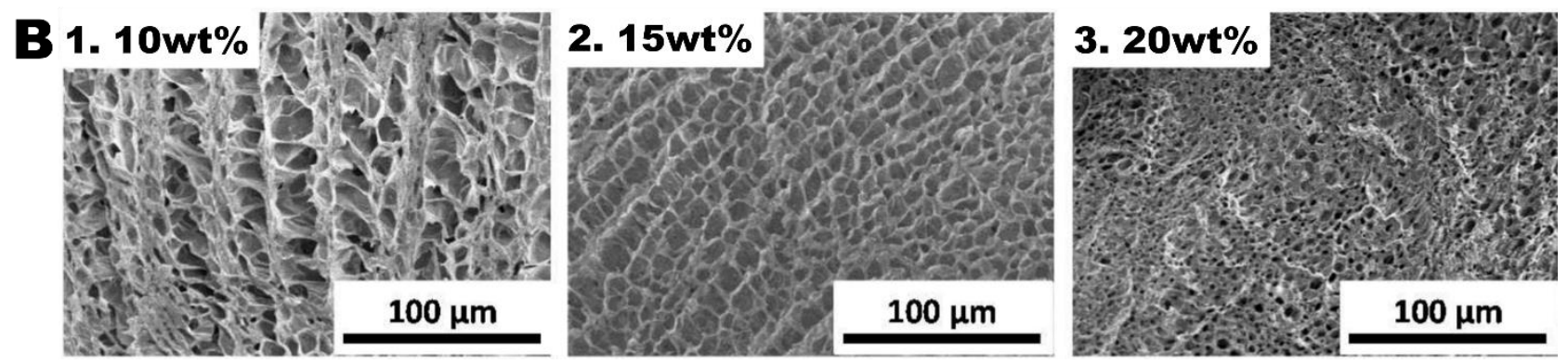

Figure 18. $\mathrm{A}$ is the preparation process of FITC-insulin/GOx-loaded oxidation-responsive hydrogel; B are the SEM images of the hydrogels prepared by Mei Zhang et al. ${ }^{44}$

The acrylic moieties hanging in the end of the main chains provided oxidation-responsive capacity. The degradation process can be classified into two approaches, affording 5 parts including phenylboronic acid, acrylic acid, thioether moiety. Phenylboronic acid was oxidized by $\mathrm{H}_{2} \mathrm{O}_{2}$, experiencing the 1,6/1,4-elimination with relatively high speed compared with the rate of thioether oxidation ${ }^{129}$. This discrepancy pointed out that the decomposition of phenylboronic acid played a major role in the degradation of hydrogels. and native substances might be able to overcome the demerits of each other and combine their strengths, affording drug carriers with ideal properties and acceptable prices. One of the typical biodegradable materials being able to combine with synthetic polymers like PEG to form hydrogel is chitosan (CS). CS is a 
natural polysaccharide composed of a series of linear copolymers of D-glucosamine and

$\mathrm{N}$-acetyl-D-glucosamine ${ }^{130}$. Due to properties such as good biocompatibility and encapsulation capacity of negatively charged proteins and peptides, CS has captured much attention in DDSs ${ }^{131,132}$. However, CS requires further structural modifications to improve its loading capacity before its application ${ }^{133-135}$. PEGylation could be considered as an acceptable method to extend blood half-life time ${ }^{136}$ and further enhance the biocompatibility of $\mathrm{CS}^{137}$. Bahman Vasheghani Farahani and coworkers fabricated a glucose-responsive semi IPN hydrogel by free radical cross-linking polymerization of CS, acrylamide (AAm) and PEG, using hydrogen peroxide as the initiator and $\mathrm{N}, \mathrm{N}^{\prime}$-methylenebisacrylamide (MBA) as the crosslinker $^{45}$. Figure 19 displays its exact structure.

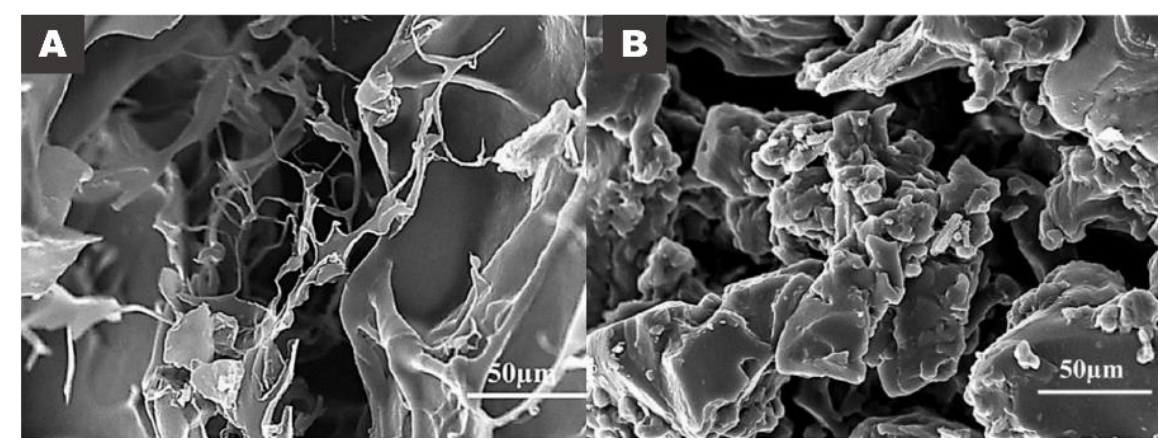

Figure 19. SEM image of chitosan semi-IPN hydrogel (A) and insulin loaded chitosan semi-IPN hydrogel (B) $)^{45}$.

The glucose-responsive property was generated mainly by GOx while CAT also contributed. Insulin was

loaded inside the hydrogel by the swelling-diffusion technique. Interestingly, increased PEG ratio led to the rise of swelling ratio, drug loading capacities and entrapment efficiency. In vitro investigation showed that per $0.1 \mathrm{~g}$ of hydrogel released 150 units of insulin under $500 \mathrm{mg} / \mathrm{dL}$ of glucose concentration, and the insulin release rate can be flexibly adjusted by altering the ratio of PEG (figure 20).

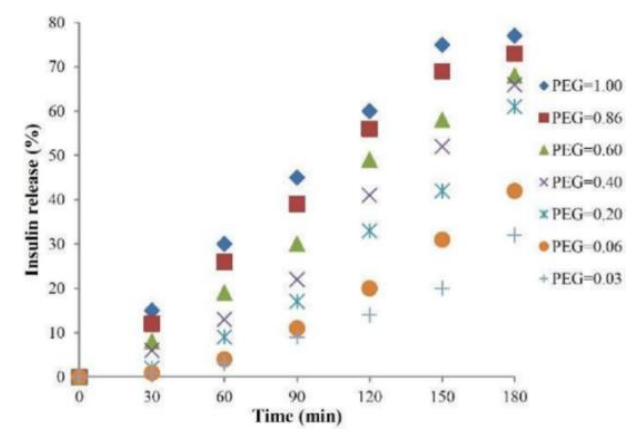

Figure 20. In vitro insulin release profiles of semi IPN hydrogels with different PEG ratio prepared by Bahman Vasheghani Farahani et al. ${ }^{45}$ 


\section{Conclusion}

Diabetes mellitus is a severe chronic metabolic disorder that causes huge economic losses and physical pain of patients. Numerous DDSs for antidiabetic drugs, including micelles, vesicles, nanoparticles, microneedles and hydrogels, have been developed to achieve efficient and convenient administration. PEG is a series of amphiphilic polymers which have been studied elaborately in DDSs. The PEG modified DDSs allow multiple physiochemical, pharmacokinetic or pharmacodynamic promotions of antidiabetic drugs such as insulin, GLP-1 analogues and others.

The large number of combinations between PEG and other natural or synthetic molecules such as chitosan and PLGA provide abundant types of carriers with diverse characters to fulfill complex demands of drugs. Generally, in the micelle systems, PEG functions as the hydrophilic tails to accelerate the self-assembly of amphiphilic copolymers to afford micelles. Besides, the hydrophilic shell formed by PEG is able to shelter micelles and loaded molecules from undesirable enzymolysis. In the meantime, the purposive modified hydrophobic heads of copolymers could endow micelles with stimuli-responsive capacity, such as the phenylboronic acidified hydrophobic heads could perceive the slight fluctuation of blood glucose level, to achieve controllable and sustainable drug release. Basically, due to the sophisticated structures, several ingredients could be loaded inside the PEGylated vesicles simultaneously. Therefore, vesicles could respond to multiple physiological stimuli to perform a promoted release profile. The highly improved drug delivery efficiency of PEGylated NPs also has been proven such as extended in vivo half-life time. In the designing and preparation of hydrogels, PEG is widely adopted as an ideal component to build these porous networks. The various combinations between PEG and other natural or artificial materials provide plenty of strategies to develop DDSs. For instance, the thermosensitive hydrogels obtained via integrating thermosensitive materials and PEG exhibit significantly extended 591 internal lifespan. 
592 This review highlights the advantages and the versatility of PEG in the designing and preparation of

593 antidiabetic DDS. Benefits like extended plasma half-life, reduced aggregation, enhanced delivery 594 efficiency, improved biocompatibility and stimuli-responsive capacity have been summarized. Predictably, 595 PEG will be continuously studied as an important chemical to develop DDS in the treatment of diabetes 596 mellitus. 


\section{Acknowledgement}

599 This work was supported by National Natural Science Foundation of China (81673296) and National Key

600 R\&D Program of China (2018YFA0901701).

601 


\section{References}

1 Sinclair, A., Saeedi, P., Kaundal, A., Karuranga, S., Malanda, B. \& Williams, R. Diabetes and global ageing among 65-99-year-old adults: Findings from the International Diabetes Federation Diabetes Atlas, 9(th) edition, Diabetes Res Clin Pract, 162 (2020) 108078, doi:10. 1016/j. diabres. 2020. 108078.

2 Saeedi, P., Salpea, P., Karuranga, S., Petersohn, I., Malanda, B., Gregg, E. W., Unwin, N., Wild, S. H. \& Williams, R. Mortality attributable to diabetes in 20-79 years old adults, 2019 estimates: Results from the International Diabetes Federation Diabetes Atlas, 9(th) edition, Diabetes Res Clin Pract, 162 (2020) 108086, doi:10. 1016/j. diabres. 2020. 108086.

L Castano, a. \& Eisenbarth, G. S. Type-I Diabetes: A Chronic Autoimmune Disease of Human, Mouse, and Rat, 8 (1990) 647-679, doi:10. 1146/annurev. iy. 08. 040190. 003243.

4 Simos, Y. V., Spyrou, K., Patila, M., Karouta, N., Stamatis, H., Gournis, D., Dounousi, E. \& Peschos, D. Trends of nanotechnology in type 2 diabetes mellitus treatment, Asian Journal of Pharmaceutical Sciences (2020), doi:10.1016/j. ajps.2020.05. 001.

5 Chatzakis, C., Goulis, D. G., Mareti, E., Eleftheriades, M., Zavlanos, A., Dinas, K. \& Sotiriadis, A. Prevention of gestational diabetes mellitus in overweight or obese pregnant women: A network meta-analysis, Diabetes Res Clin Pract, 158 (2019) 107924, doi:10.1016/j. diabres. 2019.107924.

Saeedi, P., Petersohn, I., Salpea, P., Malanda, B., Karuranga, S., Unwin, N., Colagiuri, S., Guariguata, L., Motala, A. A., Ogurtsova, K., Shaw, J. E., Bright, D., Williams, R. \& Committee, I. D. F. D. A. Global and regional diabetes prevalence estimates for 2019 and projections for 2030 and 2045: Results from the International Diabetes Federation Diabetes Atlas, 9(th) edition, Diabetes Res Clin Pract, 157 (2019) 107843, doi:10. 1016/j. diabres. 2019. 107843.

7 Zuo, X., Dong, Z., Zhang, P., Zhang, P., Chang, G., Xiang, Q., Zhu, X., Zhou, J., Qiao, C., Yang, Y., Qin, Y. \& Lou, P. Effect of cognitive behavioral therapy on sleep disturbances and quality of life among adults with type 2 diabetes mellitus: A randomized controlled trial, Nutrition, Metabolism and Cardiovascular Diseases (2020), doi:10.1016/j. numecd. 2020.06.024.

8 Razaz, J. M., Rahmani, J., Varkaneh, H. K., Thompson, J., Clark, C. \& Abdulazeem, H. M. The health effects of medical nutrition therapy by dietitians in patients with diabetes: A systematic review and meta-analysis: Nutrition therapy and diabetes, Prim Care Diabetes, 13 (2019) 399-408, doi:10. 1016/j. pcd. 2019. 05. 001.

9 Reyes-García, R., Moreno-Pérez, Ó., Tejera-Pérez, C., Fernández-García, D., Bellido-Castañeda, V., López de la Torre Casares, M., Rozas-Moreno, P., Fernández-García, J. C., Marco Martínez, A., Escalada-San Martín, J., Gargallo-Fernández, M. , Botana-López, M. , López-Fernández, J. , González-Clemente, J. M., Jódar-Gimeno, E. \& Mezquita-Raya, P. A comprehensive approach to type 2 diabetes mellitus - A recommendation document, Endocrinología, Diabetes y Nutrición (English ed.), 66 (2019) 443-458, doi:10. 1016/j. endien. 2018. 10. 013.

10 Harris, J. M., Martin, N. E. \& Modi, M. Pegylation: a novel process for modifying pharmacokinetics, Clin Pharmacokinet, 40 (2001) 539-551, doi:10.2165/00003088-200140070-00005.

11 D’Souza A, A. \& Shegokar, R. Polyethylene glycol (PEG) : a versatile polymer for pharmaceutical applications, Expert Opin Drug Deliv, 13 (2016) 1257-1275, doi:10. 1080/17425247.2016. 1182485.

Li, W., Zhan, P., De Clercq, E., Lou, H. \& Liu, X. Current drug research on PEGylation with small molecular agents, Progress in Polymer Science, 38 (2013) 421-444, doi:10. 1016/j. progpolymsci. 2012.07. 006.

13 Kolate, A., Baradia, D., Patil, S., Vhora, I., Kore, G. \& Misra, A. PEG - a versatile conjugating ligand for drugs and drug delivery systems, J Control Release, 192 (2014) 67-81, doi:10. 1016/j. jconre1. 2014. 06. 046.

14 Wang, J. Z., You, M. L., Ding, Z. Q. \& Ye, W. B. A review of emerging bone tissue engineering via PEG 
con jugated biodegradable amphiphilic copolymers, Mater Sci Eng C Mater Biol Appl, 97 (2019) 1021-1035, doi:10. 1016/j. msec. 2019.01. 057.

Mishra, P., Nayak, B. \& Dey, R. K. PEGylation in anti-cancer therapy: An overview, Asian Journal of Pharmaceutical Sciences, 11 (2016) 337-348, doi:10.1016/j. ajps. 2015. 08. 011.

Jacob, J., Haponiuk, J. T., Thomas, S. \& Gopi, S. Biopolymer based nanomaterials in drug delivery systems: A review, Materials Today Chemistry, 9 (2018) 43-55, doi:10.1016/j. mtchem. 2018. 05. 002.

Fuks, G., Mayap Talom, R. \& Gauffre, F. Biohybrid block copolymers: towards functional micelles and vesicles, Chem Soc Rev, 40 (2011) 2475-2493, doi:10.1039/c0cs00085j. Vesicles Integrated with Transcutaneous Patches for Glucose-Mediated Insulin Delivery, ACS Nano, 11 (2017) 613-620, doi:10. 1021/acsnano. 6b06892.

Morcol, T., Nagappan, P., Nerenbaum, L., Mitche11, A. \& Be11, S. J. Calcium phosphate-PEG-insulin-casein (CAPIC) particles as oral delivery systems for insulin, Int J Pharm, 277 (2004) 91-97, doi:10. 1016/j. i jpharm. 2003. 07.015.

Wang, W., Liao, L., Zhang, X., Lei, F., Zhang, Y., Liu, G. \& Xie, W. An Intelligent Nanoscale Insulin Delivery System, Molecules (Basel, Switzerland), 23 (2018), doi:10.3390/molecules23112945.

Yu, J., Zhang, Y., Yan, J., Kahkoska, A. R. \& Gu, Z. Advances in bioresponsive closed-1oop drug delivery systems, Int J Pharm, 544 (2018) 350-357, doi:10. 1016/j. i jpharm. 2017.11.064.

Zhao, L., Ding, J., Xiao, C., He, P., Tang, Z., Pang, X., Zhuang, X. \& Chen, X. Glucose-sensitive polypeptide micelles for self-regulated insulin release at physiological pH, Journal of Materials Chemistry, 22 (2012), doi:10. 1039/c2jm31040f.

Fang, X., Yang, T., Wang, L., Yu, J., Wei, X., Zhou, Y., Wang, C. \& Liang, W. Nano-cage-mediated refolding of insulin by PEG-PE micelle, Biomaterials, $77 \quad$ (2016) 139-148, doi:10. 1016/j. biomaterials. 2015. 11. 007.

Wu, G., Li, C., Liu, X., Lv, J., Ding, Y., Liu, Y., Liu, Y., Huang, F., Shi, L., An, Y. \& Ma, R. Glucose-responsive complex micelles for self-regulated delivery of insulin with effective protection of insulin and enhanced hypoglycemic activity in vivo, Colloids Surf B Biointerfaces, 180 (2019) 376-383, doi:10. 1016/j. colsurfb. 2019. 05. 003.

Ma, R., Yang, H., Li, Z., Liu, G., Sun, X., Liu, X., An, Y. \& Shi, L. Phenylboronic acid-based complex micelles with enhanced glucose-responsiveness at physiological pH by complexation with glycopolymer, Biomacromolecules, 13 (2012) 3409-3417, doi:10. 1021/bm3012715.

Wang, J., Li, S., Chen, T., Xian, W., Zhang, H., Wu, L., Zhu, W. \& Zeng, Q. Nanoscale cationic micelles of amphiphilic copolymers based on star-shaped PLGA and PEI cross-linked PEG for protein delivery application, J Mater Sci Mater Med, 30 (2019) 93, doi:10. 1007/s10856-019-6294-y.

Rastogi, R., Anand, S. \& Koul, V. Evaluation of pharmacological efficacy of 'insulin-surfoplex' encapsulated polymer vesicles, Int J Pharm, 373 (2009) 107-115, doi:10. 1016/j. i jpharm. 2009.01.022. Tai, W., Mo, R., Di, J., Subramanian, V., Gu, X., Buse, J. B. \& Gu, Z. Bio-inspired synthetic nanovesicles for glucose-responsive release of insulin, Biomacromolecules, $15 \quad$ (2014) 3495-3502, doi:10. 1021/bm500364a.

Yu, J., Qian, C., Zhang, Y., Cui, Z., Zhu, Y., Shen, Q., Ligler, F. S., Buse, J. B. \& Gu, Z. Hypoxia and H202 Dual-Sensitive Vesicles for Enhanced Glucose-Responsive Insulin Delivery, Nano Lett, 17 (2017) 733-739, doi:10. 1021/acs. nanolett.6b03848.

Kim, A., Yun, M. 0., Oh, Y. K., Ahn, W. S. \& Kim, C. K. Pharmacodynamics of insulin in polyethylene glycol-coated liposomes, Int J Pharm, 180 (1999) 75-81, doi:10. 1016/s0378-5173 (98) 00408-6.

31 Haggag, Y., Abde1-Wahab, Y., 0jo, 0., Osman, M. , E1-Gizawy, S., E1-Tanani, M. , Faheem, A. \& McCarron, P. Preparation and in vivo evaluation of insulin-loaded biodegradable nanoparticles prepared from diblock copolymers of PLGA and PEG, Int J Pharm, 499 (2016) 236-246, doi:10. 1016/j. i jpharm. 2015. 12.063. Malathi, S., Nandhakumar, P., Pandiyan, V., Webster, T. J. \& Balasubramanian, S. Novel PLGA-based nanoparticles for the oral delivery of insulin, Int J Nanomedicine, 10 (2015) 2207-2218, 
doi : 10. 2147/IJN. S67947.

Shi, Y., Sun, X., Zhang, L., Sun, K., Li, K., Li, Y. \& Zhang, Q. Fc-modified exenatide-loaded nanoparticles for oral delivery to improve hypoglycemic effects in mice, Sci Rep, 8 (2018) 726, doi : 10. 1038/s41598-018-19170-y.

Chopra, S., Bertrand, N., Lim, J. M., Wang, A., Farokhzad, 0. C. \& Karnik, R. Design of Insulin-Loaded Nanoparticles Enabled by Multistep Control of Nanoprecipitation and Zinc Chelation, ACS Appl Mater Interfaces, 9 (2017) 11440-11450, doi:10. 1021/acsami.6b16854.

Yu, F., Li, Y., Liu, C. S., Chen, Q., Wang, G. H., Guo, W., Wu, X. E., Li, D. H., Wu, W. D. \& Chen, X. D. Enteric-coated capsules filled with mono-disperse micro-particles containing PLGA-1ipid-PEG nanoparticles for oral delivery of insulin, Int J Pharm, 484 (2015) 181-191, doi:10. 1016/j. i jpharm. 2015. 02. 055.

Nie, T., He, Z., Zhou, Y., Zhu, J., Chen, K., Liu, L., Leong, K. W., Mao, H. Q. \& Chen, Y. Surface Coating Approach to Overcome Mucosal Entrapment of DNA Nanoparticles for Oral Gene Delivery of Glucagon-1ike Peptide 1, ACS Appl Mater Interfaces, 11 (2019) 29593-29603, doi:10. 1021/acsami. 9 b10294.

Tong, F. Preparation of exenatide-loaded linear poly(ethylene glycol)-brush poly(1-lysine) block copolymer: potential implications on diabetic nephropathy, Int J Nanomedicine, 12 (2017) 4663-4678, doi : 10. 2147/IJN. S136646.

Zhang, L., Shi, Y., Song, Y., Sun, X., Zhang, X., Sun, K. \& Li, Y. The use of low molecular weight protamine to enhance oral absorption of exenatide, Int J Pharm, 547 (2018) 265-273, doi:10. 1016/j. i jpharm. 2018. 05. 055.

Jain, S. \& Saraf, S. Repaglinide-loaded long-circulating biodegradable nanoparticles: rational approach for the management of type 2 diabetes mellitus, J Diabetes, 1 (2009) 29-35, doi:10. 1111/j. 1753-0407. 2008. 00001. x.

Sharma, D. \& Singh, J. Long-term glycemic control and prevention of diabetes complications in vivo using oleic acid-grafted-chitosanzinc-insulin complexes incorporated in thermosensitive copolymer, J Control Release, 323 (2020) 161-178, doi:10.1016/j. jconre1.2020. 04.012.

Chen, Y., Luan, J., Shen, W., Lei, K., Yu, L. \& Ding, J. Injectable and Thermosensitive Hydroge1 Containing Liraglutide as a Long-Acting Antidiabetic System, ACS Appl Mater Interfaces, 8 (2016) 30703-30713, doi:10. 1021/acsami. 6b09415.

Zhuang, Y., Yang, X., Li, Y., Chen, Y., Peng, X., Yu, L. \& Ding, J. Sustained Release Strategy Designed for Lixisenatide Delivery to Synchronously Treat Diabetes and Associated Complications, ACS Appl Mater Interfaces, 11 (2019) 29604-29618, doi:10.1021/acsami.9b10346.

Wang, P., Zhuo, X., Chu, W. \& Tang, X. Exenatide-loaded microsphere/thermosensitive hydrogel long-acting delivery system with high drug bioactivity, Int J Pharm, 528 (2017) 62-75, doi:10. 1016/j. i jpharm. 2017.05. 069.

Zhang, M., Song, C. C., Du, F. S. \& Li, Z. C. Supersensitive Oxidation-Responsive Biodegradable PEG Hydrogels for Glucose-Triggered Insulin Delivery, ACS Appl Mater Interfaces, 9 (2017) 25905-25914, doi:10. 1021/acsami. 7b08372.

45 Farahani, B. V., Ghasemzaheh, H. \& Afraz, S. Intelligent semi-IPN chitosan - PEG-PAAm hydrogel for closed-loop insulin delivery and kinetic modeling, RSC Advances, 6 (2016) 26590-26598, doi:10. 1039/c5ra28188a.

46 Zhang, S., Xin, P., Ou, Q., Hollett, G., Gu, Z. \& Wu, J. Poly (ester amide)-based hybrid hydrogels for efficient transdermal insulin delivery, J Mater Chem B, 6 (2018) 6723-6730, doi:10.1039/c8tb01466c.

Tanner, P., Baumann, P., Enea, R., Onaca, 0., Palivan, C. \& Meier, W. Polymeric vesicles: from drug carriers to nanoreactors and artificial organelles, Accounts of chemical research, 44 (2011) 1039-1049, doi:10. 1021/ar200036k.

48 Jin, X., Zhu, D. D., Chen, B. Z., Ashfaq, M. \& Guo, X. D. Insulin delivery systems combined with microneedle technology, Adv Drug Deliv Rev, 127 (2018) 119-137, doi:10.1016/j. addr. 2018. 03.011. 
management, Indian journal of pharmacology, 51 (2019) 4-10, doi:10.4103/ijp. IJP_16_18.

Wu, Q., Wang, L., Yu, H., Wang, J. \& Chen, Z. Organization of glucose-responsive systems and their properties, Chem Rev, 111 (2011) 7855-7875, doi:10. 1021/cr200027j.

Kuivila, H. G., Keough, A. H. \& Soboczenski, E. J. ARENEBORONATES FROM DIOLS AND POLYOLS1, The Journal of Organic Chemistry, 19 (1954) 780-783, doi:10. 1021/jo01370a013.

Ma, R. \& Shi, L. Phenylboronic acid-based glucose-responsive polymeric nanoparticles: synthesis and applications in drug delivery, Polym. Chem., 5 (2014) 1503-1518, doi:10. 1039/c3py01202f.

Shen, D., Yu, H., Wang, L., Khan, A., Haq, F., Chen, X., Huang, Q. \& Teng, L. Recent progress in design and preparation of glucose-responsive insulin delivery systems, J Control Release, 321 (2020) 236-258, doi:10. 1016/j. jconre1. 2020. 02. 014.

Yang, H., Sun, X., Liu, G., Ma, R., Li, Z., An, Y. \& Shi, L. Glucose-responsive complex micelles for self-regulated release of insulin under physiological conditions, Soft Matter, 9 (2013), doi:10. 1039/c3sm51538a.

Wang, J., Ye, Y., Yu, J., Kahkoska, A. R., Zhang, X., Wang, C., Sun, W., Corder, R. D., Chen, Z., Khan, S. A., Buse, J. B. \& Gu, Z. Core-Shell Microneedle Gel for Self-Regulated Insulin Delivery, ACS Nano, 12 (2018) 2466-2473, doi:10.1021/acsnano. 7b08152.

Torchilin, V. P. Micellar nanocarriers: pharmaceutical perspectives, Pharm Res, 24 (2007) 1-16, doi:10. 1007/s11095-006-9132-0.

Wang, J., Hu, X. \& Xiang, D. Nanoparticle drug delivery systems: an excellent carrier for tumor peptide vaccines, Drug Deliv, 25 (2018) 1319-1327, doi:10. 1080/10717544.2018. 1477857.

Quijia Quezada, C., Azevedo, C. S., Charneau, S., Santana, J. M., Chorilli, M., Carneiro, M. B. \& Bastos, I. M. D. Advances in nanocarriers as drug delivery systems in Chagas disease, Int J Nanomedicine, 14 (2019) 6407-6424, doi:10.2147/IJN. S206109.

Edgar, J. Y. C. \& Wang, H. Introduction for Design of Nanoparticle Based Drug Delivery Systems, Current pharmaceutical design, 23 (2017) 2108-2112, doi:10.2174/1381612822666161025154003.

Adams, M. L., Lavasanifar, A. \& Kwon, G. S. Amphiphilic block copolymers for drug delivery, J Pharm Sci, 92 (2003) 1343-1355, doi:10. 1002/jps. 10397.

61 Khoee, S. \& Rahmatolahzadeh, R. Synthesis and characterization of $\mathrm{pH}$-responsive and folated nanoparticles based on self-assembled brush-like PLGA/PEG/AEMA copolymer with targeted cancer therapy properties: A comprehensive kinetic study, Eur J Med Chem, 50 (2012) 416-427, doi:10. 1016/j. ejmech. 2012.02. 027.

Ashok, B., Rubinstein, I., Tsueshita, T. \& Onyukse1, H. Effects of peptide molecular mass and PEG chain length on the vasoreactivity of VIP and PACAP(1-38) in pegylated phospholipid micelles, Peptides, 25 (2004) 1253-1258, doi:10.1016/j. peptides. 2004.05. 013.

Schoch, R. L., Emilsson, G., Dahlin, A. B. \& Lim, R. Y. H. Protein exclusion is preserved by temperature sensitive PEG brushes, Polymer, 132 (2017) 362-367, doi:10.1016/j. polymer.2017. 10. 063.

Akbarian, M., Ghasemi, Y., Uversky, V. N. \& Yousefi, R. Chemical modifications of insulin: Finding a compromise between stability and pharmaceutical performance, Int J Pharm, 547 (2018) 450-468, doi:10. 1016/j. i jpharm. 2018. 06. 023.

Fineberg, S. E., Kawabata, T. T., Finco-Kent, D., Fountaine, R. J., Finch, G. L. \& Krasner, A. S. Immunological responses to exogenous insulin, Endocr Rev, 28 (2007) 625-652, doi:10. 1210/er. 2007-0002. Siposova, K., Pospiskova, K., Bednarikova, Z., Safarik, I., Safarikova, M., Kubovcikova, M., Kopcansky, P. \& Gazova, Z. The molecular mass of dextran used to modify magnetite nanoparticles affects insulin amyloid aggregation, Journal of Magnetism and Magnetic Materials, 427 (2017) 48-53, doi:10. 1016/j. jmmm. 2016. 10. 083.

Lee, E. J., Lee, N. K. \& Kim, I. S. Bioengineered protein-based nanocage for drug delivery, Adv Drug Deliv Rev, 106 (2016) 157-171, doi:10.1016/j. addr. 2016.03.002.

Kapoor, D. N., Bhatia, A., Kaur, R., Sharma, R., Kaur, G. \& Dhawan, S. PLGA: a unique polymer for drug delivery, Ther Deliv, 6 (2015) 41-58, doi:10.4155/tde. 14.91. 
Ding, D. \& Zhu, Q. Recent advances of PLGA micro/nanoparticles for the delivery of biomacromolecular therapeutics, Mater Sci Eng C Mater Biol App1, 92 (2018) 1041-1060, doi:10. 1016/j. msec. 2017. 12.036. Danhier, F., Ansorena, E., Silva, J. M., Coco, R., Le Breton, A. \& Preat, V. PLGA-based nanoparticles: an overview of biomedical applications, J Control Release, 161 (2012) 505-522, doi:10. 1016/j. jconre1. 2012.01. 043.

Kang, H. C., Lee, J. E. \& Bae, Y. H. Nanoscaled buffering zone of charged (PLGA)n-b-bPEI micelles in acidic microclimate for potential protein delivery application, J Control Release, 160 (2012) 440-450, doi:10. 1016/j. jconrel. 2012. 02. 024.

Zhang, Y., Lin, L., Liu, L., Liu, F., Maruyama, A., Tian, H. \& Chen, X. Ionic-crosslinked polysaccharide/PEI/DNA nanoparticles for stabilized gene delivery, Carbohydr Polym, 201 (2018) 246-256, doi:10. 1016/j. carbpo1. 2018. 08. 063.

Dong, X., Tian, H., Chen, L., Chen, J. \& Chen, X. Biodegradable mPEG-b-P(MCC-g-0EI) copolymers for efficient gene delivery, J Control Release, 152 (2011) 135-142, doi:10. 1016/j. jconre1.2011.03.025. Huang, F. W. , Wang, H. Y., Li, C., Wang, H. F., Sun, Y. X., Feng, J., Zhang, X. Z. \& Zhuo, R. X. PEGylated PEI-based biodegradable polymers as non-viral gene vectors, Acta Biomater, 6 (2010) 4285-4295, doi:10. 1016/j. actbio. 2010. 06. 016.

Vader, P., Mol, E. A., Pasterkamp, G. \& Schiffelers, R. M. Extracellular vesicles for drug delivery, Adv Drug Deliv Rev, 106 (2016) 148-156, doi:10. 1016/j. addr.2016. 02. 006.

Ha, D., Yang, N. \& Nadithe, V. Exosomes as therapeutic drug carriers and delivery vehicles across biological membranes: current perspectives and future challenges, Acta pharmaceutica Sinica. B, 6 (2016) 287-296, doi:10. 1016/j. apsb. 2016. 02.001.

Surman, M. , Drożdż, A., Stępień, E. \& Przybyło, M. Extracellular Vesicles as Drug Delivery Systems Methods of Production and Potential Therapeutic Applications, Current pharmaceutical design, 25 (2019) 132-154, doi:10.2174/1381612825666190306153318.

Kim, M. S., Haney, M. J., Zhao, Y., Yuan, D., Deygen, I., Klyachko, N. L., Kabanov, A. V. \& Batrakova, E. V. Engineering macrophage-derived exosomes for targeted paclitaxel delivery to pulmonary metastases: in vitro and in vivo evaluations, Nanomedicine, 14 (2018) 195-204, doi:10. 1016/j. nano. 2017.09.011. Liu, Y., Du, J., Yan, M., Lau, M. Y., Hu, J., Han, H., Yang, 0. 0., Liang, S., Wei, W., Wang, H., Li, J., Zhu, X., Shi, L., Chen, W., Ji, C. \& Lu, Y. Biomimetic enzyme nanocomplexes and their use as antidotes and preventive measures for alcohol intoxication, Nat Nanotechnol, 8 (2013) 187-192, doi : 10. 1038/nnano. 2012. 264.

Traitel, T., Cohen, Y. \& Kost, J. Characterization of glucose-sensitive insulin release systems in simulated in vivo conditions, Biomaterials, 21 (2000) 1679-1687, doi:10. 1016/s0142-9612(00)00050-8.

Zhang, K. \& Wu, X. Y. Modulated insulin permeation across a glucose-sensitive polymeric composite membrane, J Control Release, 80 (2002) 169-178, doi:10. 1016/s0168-3659 (02) 00024-x.

Zhao, L., Xiao, C., Wang, L., Gai, G. \& Ding, J. Glucose-sensitive polymer nanoparticles for self-regulated drug delivery, Chem Commun (Camb), 52 (2016) 7633-7652, doi:10. 1039/c6cc02202b. Kochhar, J. S., Lim, W. X., Zou, S., Foo, W. Y., Pan, J. \& Kang, L. Microneedle integrated transdermal patch for fast onset and sustained delivery of lidocaine, Mol Pharm, 10 (2013) 4272-4280, doi:10. 1021/mp400359w.

Ito, Y., Nakahigashi, T., Yoshimoto, N., Ueda, Y., Hamasaki, N. \& Takada, K. Transdermal insulin application system with dissolving microneedles, Diabetes Technol Ther, 14 (2012) 891-899, doi:10. 1089/dia. 2012. 0096.

McAllister, D. V., Wang, P. M., Davis, S. P., Park, J. H., Canatella, P. J., Allen, M. G. \& Prausnitz, M. R. Microfabricated needles for transdermal delivery of macromolecules and nanoparticles: fabrication methods and transport studies, Proc Natl Acad Sci U S A, 100 (2003) 13755-13760, doi:10. 1073/pnas. 2331316100 .

Zhang, Y., Yu, J., Kahkoska, A. R., Wang, J., Buse, J. B. \& Gu, Z. Advances in transdermal insulin delivery, Adv Drug Deliv Rev, 139 (2019) 51-70, doi:10. 1016/j. addr. 2018. 12. 006. 
Donnelly, R. F., Raj Singh, T. R. \& Woolfson, A. D. Microneedle-based drug delivery systems: microfabrication, drug delivery, and safety, Drug Deliv, 17 (2010) 187-207, doi:10. 3109/10717541003667798.

88 Nuxoll, E. E. \& Siegel, R. A. BioMEMS devices for drug delivery, IEEE Eng Med Biol Mag, 28 (2009) 31-39, doi:10. 1109/MEMB. 2008. 931014.

Napoli, A., Valentini, M., Tirelli, N., Muller, M. \& Hubbe1l, J. A. Oxidation-responsive polymeric vesicles, Nat Mater, 3 (2004) 183-189, doi:10.1038/nmat1081.

Huo, M. , Yuan, J., Tao, L. \& Wei, Y. Redox-responsive polymers for drug delivery: from molecular design to applications, Polym. Chem., 5 (2014) 1519-1528, doi:10.1039/c3py01192e.

91 Yu, J., Zhang, Y., Ye, Y., DiSanto, R., Sun, W., Ranson, D., Ligler, F. S., Buse, J. B. \& Gu, Z. Microneedle-array patches loaded with hypoxia-sensitive vesicles provide fast glucose-responsive insulin delivery, Proc Natl Acad Sci U S A, 112 (2015) 8260-8265, doi:10. 1073/pnas. 1505405112.

Krohn, K. A., Link, J. M. \& Mason, R. P. Molecular imaging of hypoxia, J Nucl Med, 49 Supp1 2 (2008) 129S-148S, doi:10.2967/jnumed. 107. 045914.

Suk, J. S., Xu, Q., Kim, N., Hanes, J. \& Ensign, L. M. PEGylation as a strategy for improving nanoparticle-based drug and gene delivery, Adv Drug Deliv Rev, 99 (2016) 28-51, doi:10. 1016/j. addr. 2015. 09. 012.

Haggag, Y. A., Faheem, A. M., Tambuwala, M. M., Osman, M. A., E1-Gizawy, S. A., 0’ Hagan, B., Irwin, N. \& McCarron, P. A. Effect of poly (ethylene glycol) content and formulation parameters on particulate properties and intraperitoneal delivery of insulin from PLGA nanoparticles prepared using the double-emulsion evaporation procedure, Pharmaceutical development and technology, 23 (2018) 370-381, doi:10. 1080/10837450. 2017. 1295066.

95 Ramachandran, R., Paul, W. \& Sharma, C. P. Synthesis and characterization of PEGylated calcium phosphate nanoparticles for oral insulin delivery, Journal of biomedical materials research. Part B, Applied biomaterials, 88 (2009) 41-48, doi:10. 1002/jbm. b. 31241.

Tomar, L., Tyagi, C., Kumar, M., Kumar, P., Singh, H., Choonara, Y. E. \& Pillay, V. In vivo evaluation of a conjugated poly(lactide-ethylene glycol) nanoparticle depot formulation for prolonged insulin delivery in the diabetic rabbit model, Int J Nanomedicine, 8 (2013) 505-520, doi:10.2147/i jn. S38011. Alavi, S. E., Cabot, P. J. \& Moyle, P. M. Glucagon-Like Peptide-1 Receptor Agonists and Strategies To Improve Their Efficiency, Mol Pharm, 16 (2019) 2278-2295, doi:10. 1021/acs. molpharmaceut. 9b00308. Holst, J. J. \& Gromada, J. Role of incretin hormones in the regulation of insulin secretion in diabetic and nondiabetic humans, Am J Physiol Endocrinol Metab, 287 (2004) E199-206, doi:10. 1152/ajpendo. 00545. 2003.

Khan, R., Tomas, A. \& Rutter, G. A. Effects on pancreatic Beta and other Islet cells of the glucose-dependent insulinotropic polypeptide, Peptides, $125 \quad$ (2020) 170201, doi:10. 1016/j. peptides. 2019. 170201.

100 DeYoung, M. B., MacCone11, L., Sarin, V., Trautmann, M. \& Herbert, P. Encapsulation of exenatide in poly-(D, L-lactide-co-glycolide) microspheres produced an investigational long-acting once-weekly formulation for type 2 diabetes, Diabetes Technol Ther, 13 (2011) 1145-1154, doi:10. 1089/dia. 2011. 0050.

101 Cullis, P. R. \& Hope, M. J. Lipid Nanoparticle Systems for Enabling Gene Therapies, Mol Ther, 25 (2017) 1467-1475, doi:10.1016/j. ymthe. 2017.03. 013.

102 Wang, X., Niu, D., Hu, C. \& Li, P. Polyethyleneimine-Based Nanocarriers for Gene Delivery, Current pharmaceutical design, 21 (2015) 6140-6156, doi:10.2174/1381612821666151027152907.

103 Pandey, A. P. \& Sawant, K. K. Polyethylenimine: A versatile, multifunctional non-viral vector for nucleic acid delivery, Mater Sci Eng C Mater Biol Appl, 68 (2016) 904-918, doi:10. 1016/j. msec. 2016.07. 066.

104 Lungwitz, U., Breunig, M., Blunk, T. \& Gopferich, A. Polyethylenimine-based non-viral gene delivery systems, Eur J Pharm Biopharm, 60 (2005) 247-266, doi:10. 1016/j. ejpb. 2004.11.011. 
Ziaee, A., Albadarin, A. B., Padrela, L., Femmer, T., 0’Reilly, E. \& Walker, G. Spray drying of pharmaceuticals and biopharmaceuticals: Critical parameters and experimental process optimization approaches, Eur J Pharm Sci, 127 (2019) 300-318, doi:10. 1016/j. ejps. 2018. 10. 026.

106 Arpagaus, C., Collenberg, A., Rutti, D., Assadpour, E. \& Jafari, S. M. Nano spray drying for encapsulation of pharmaceuticals, Int J Pharm, 546 (2018) 194-214, doi:10. 1016/j. ijpharm. 2018. 05.037.

107 Wanning, S., Suverkrup, R. \& Lamprecht, A. Pharmaceutical spray freeze drying, Int J Pharm, 488 (2015) 136-153, doi:10. 1016/j. i jpharm. 2015. 04. 053.

108 Grigoras, A. G. Polymer-lipid hybrid systems used as carriers for insulin delivery, Nanomedicine, 13 (2017) 2425-2437, doi:10. 1016/j. nano. 2017. 08. 005.

109 Dreiss, C. A. Hydrogel design strategies for drug delivery, Current Opinion in Colloid \& Interface Science, 48 (2020) 1-17, doi:10.1016/j. cocis. 2020.02. 001.

110 Li, K., Yu, L., Liu, X., Chen, C., Chen, Q. \& Ding, J. A long-acting formulation of a polypeptide drug exenatide in treatment of diabetes using an injectable block copolymer hydrogel, Biomaterials, 34 (2013) 2834-2842, doi:10.1016/j. biomaterials. 2013.01.013.

111 Chen, Y., Li, Y., Shen, W., Li, K., Yu, L., Chen, Q. \& Ding, J. Controlled release of liraglutide using thermogelling polymers in treatment of diabetes, Sci Rep, 6 (2016) 31593, doi:10. 1038/srep31593.

112 Choi, S., Baudys, M. \& Kim, S. W. Control of blood glucose by novel GLP-1 delivery using biodegradable triblock copolymer of PLGA-PEG-PLGA in type 2 diabetic rats, Pharm Res, 21 (2004) 827-831, doi:10. 1023/b:pham. 0000026435. 27086. 94.

113 Yu, L., Li, K., Liu, X., Chen, C., Bao, Y., Ci, T., Chen, Q. \& Ding, J. In vitro and in vivo evaluation of a once-weekly formulation of an antidiabetic peptide drug exenatide in an injectable thermogel, J Pharm Sci, 102 (2013) 4140-4149, doi:10. 1002/jps. 23735.

114 Huynh, D. P., Im, G. J., Chae, S. Y., Lee, K. C. \& Lee, D. S. Controlled release of insulin from pH/temperature-sensitive injectable pentablock copolymer hydrogel, J Control Release, 137 (2009) 20-24, doi:10. 1016/j. jconre1. 2009. 02. 021.

115 Oak, M. \& Singh, J. Chitosan-zinc-insulin complex incorporated thermosensitive polymer for controlled delivery of basal insulin in vivo, J Control Release, 163 (2012) 145-153, doi:10. 1016/j. jconrel. 2012.07. 035.

116 Wang, Q., Zuo, Z., Cheung, C. K. C. \& Leung, S. S. Y. Updates on thermosensitive hydrogel for nasal, ocular and cutaneous delivery, Int J Pharm, 559 (2019) 86-101, doi:10.1016/j. ijpharm. 2019.01.030.

117 Qureshi, D., Nayak, S. K., Maji, S., Anis, A., Kim, D. \& Pal, K. Environment sensitive hydrogels for drug delivery applications, European Polymer Journal, 120 (2019), doi:10. 1016/j. eurpolymj. 2019. 109220.

118 Norouzi, M. , Nazari, B. \& Miller, D. W. Injectable hydrogel-based drug delivery systems for local cancer therapy, Drug Discov Today, 21 (2016) 1835-1849, doi:10. 1016/j. drudis. 2016. 07. 006.

119 Dutta, K., Das, R., Ling, J., Monibas, R. M., Carballo-Jane, E., Kekec, A., Feng, D. D., Lin, S., Mu, J., Saklatvala, R., Thayumanavan, S. \& Liang, Y. In Situ Forming Injectable Thermoresponsive Hydrogels for Controlled Delivery of Biomacromolecules, ACS omega, $5 \quad$ (2020) 17531-17542, doi: 10. 1021/acsomega. 0c02009.

120 Vilsboll, T. Liraglutide: a once-daily GLP-1 analogue for the treatment of type 2 diabetes mellitus, Expert Opin Investig Drugs, 16 (2007) 231-237, doi:10. 1517/13543784. 16.2.231.

121 Ryan, G. J., Foster, K. T. \& Jobe, L. J. Review of the therapeutic uses of liraglutide, Clin Ther, 33 (2011) 793-811, doi:10. 1016/j. clinthera. 2011.06. 004.

122 Muller, T. D., Finan, B., Bloom, S. R., D’Alessio, D., Drucker, D. J., Flatt, P. R., Fritsche, A., Gribble, F., Grill, H. J., Habener, J. F., Holst, J. J., Langhans, W., Meier, J. J., Nauck, M. A. , Perez-Tilve, D., Pocai, A., Reimann, F., Sandoval, D. A., Schwartz, T. W., Seeley, R. J., Stemmer, K., Tang-Christensen, M., Woods, S. C., DiMarchi, R. D. \& Tschop, M. H. Glucagon-1ike peptide 1 (GLP-1), Mol Metab, 30 (2019) 72-130, doi:10. 1016/j. molmet. 2019. 09. 010. 
tubular ectopic lipid deposition in rats with diabetic nephropathy by inhibiting lipid synthesis and promoting lipolysis, Pharmacol Res, 156 (2020) 104778, doi:10. 1016/j. phrs. 2020. 104778.

Vilsbol1, T., Brock, B., Perrild, H., Levin, K., Lervang, H. H., Kolendorf, K., Krarup, T., Schmitz, 0., Zdravkovic, M., Le-Thi, T. \& Madsbad, S. Liraglutide, a once-daily human GLP-1 analogue, improves pancreatic B-cell function and arginine-stimulated insulin secretion during hyperglycaemia in patients with Type 2 diabetes mellitus, Diabet Med, 25 (2008) 152-156, doi:10.1111/j. 1464-5491.2007. 02333.x.

125 Russe11-Jones, D. Molecular, pharmacological and clinical aspects of liraglutide, a once-daily human GLP-1 analogue, Mol Cell Endocrinol, 297 (2009) 137-140, doi:10.1016/j. mce. 2008.11. 018.

126 Newsome, J. S. Lixisenatide: A New Option for Managing Type 2 Diabetes, Journal of Pharmacy Technology, 33 (2017) 195-203, doi:10. 1177/8755122517711958.

127 Varin, E. M., McLean, B. A. \& Lovshin, J. A. Glucagon-Like Peptide-1 Receptor Agonists in Adult Patients With Type 2 Diabetes: Review of Cardiovascular Outcome Trials, Can J Diabetes, 44 (2020) 68-77, doi:10. 1016/j. jcjd. 2019. 08. 011.

128 Meng, L., Li, X. Y., Shen, L. \& Ji, H. F. Type 2 Diabetes Mellitus Drugs for Alzheimer's Disease: Current Evidence and Therapeutic Opportunities, Trends Mol Med, 26 (2020) 597-614, doi:10. 1016/j. molmed. 2020. 02. 002.

129 Song, C. -C., Ji, R., Du, F. -S. \& Li, Z. -C. Oxidation-Responsive Poly(amino ester)s Containing Arylboronic Ester and Self-Immolative Motif: Synthesis and Degradation Study, Macromolecules, 46 (2013) 8416-8425, doi:10.1021/ma401656t.

130 Sorlier, P., Denuziere, A., Viton, C. \& Domard, A. Relation between the degree of acetylation and the electrostatic properties of chitin and chitosan, Biomacromolecules, 2 (2001) 765-772, doi:10. 1021/bm015531+.

131 Gong, C., Qi, T., Wei, X., Qu, Y., Wu, Q., Luo, F. \& Qian, Z. Thermosensitive Polymeric Hydrogels As Drug Delivery Systems, Current Medicinal Chemistry, 20 (2013) 79-94.

132 Almeida, A., Arau jo, M., Novoa-Carballal, R., Andrade, F., Goncalves, H., Reis, R. L., Lucio, M., Schwartz, S., Jr. \& Sarmento, B. Novel amphiphilic chitosan micelles as carriers for hydrophobic anticancer drugs, Mater Sci Eng C Mater Biol Appl, 112 (2020) 110920, doi:10. 1016/j. msec. 2020. 110920.

133 Zhang, C., Ding, Y., Yu, L. L. \& Ping, Q. Polymeric micelle systems of hydroxycamptothecin based on amphiphilic N-alkyl-N-trimethyl chitosan derivatives, Colloids Surf B Biointerfaces, 55 (2007) 192-199, doi:10. 1016/j. colsurfb. 2006. 11. 031.

134 Zhou, Y. Y., Du, Y. Z., Wang, L., Yuan, H., Zhou, J. P. \& Hu, F. Q. Preparation and pharmacodynamics of stearic acid and poly (lactic-co-glycolic acid) grafted chitosan oligosaccharide micelles for 10-hydroxycamptothecin, Int J Pharm, 393 (2010) 143-151, doi:10. 1016/j. i jpharm. 2010. 04. 025.

135 Zhang, M., Li, X. H., Gong, Y. D., Zhao, N. M. \& Zhang, X. F. Properties and biocompatibility of chitosan films modified by blending with PEG, Biomaterials, 23 (2002) 2641-2648, doi:10. 1016/s0142-9612(01) 00403-3.

136 Sugimoto, M., Morimoto, M., Sashiwa, H., Saimoto, H. \& Shigemasa, Y. Preparation and characterization of water-soluble chitin and chitosan derivatives, Carbohydrate Polymers, 36 (1998) 49-59, doi:Doi 10. 1016/S0144-8617 (97) 00235-X.

137 Prego, C., Torres, D., Fernandez-Megia, E., Novoa-Carballal, R., Quinoa, E. \& Alonso, M. J. Chitosan-PEG nanocapsules as new carriers for oral peptide delivery. Effect of chitosan pegylation degree, J Control Release, 111 (2006) 299-308, doi:10.1016/j. jconre1.2005. 12. 015. 